\title{
ARGENTINE REPUBLIC
}

DEPARTMENT OF AGRICULTURE

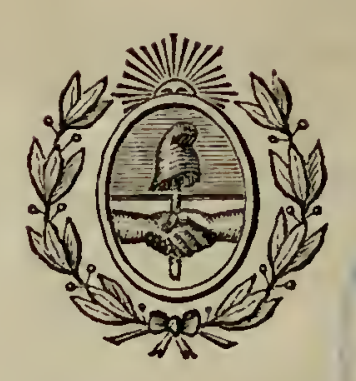

\section{CATALOGUE}

OF

\section{CEREALS AND GRAIN SAMPLES}

COLLECTEI) FROM ALL PARTS OF THE REPUBLIC

$$
\text { UNDER THE SUPERVISION }
$$

OF THE

"SALA DE COMERCIO ONCE DE SEPTIEMBRE"

BUENOS AIRES 


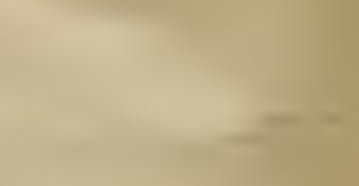

$x^{2}$ 


\begin{tabular}{|c|c|c|c|c|c|c|c|}
\hline \multirow{2}{*}{ No. } & \multirow{2}{*}{\multicolumn{2}{|c|}{ PRODUCT }} & \multirow{2}{*}{ EXHIBITOR } & \multicolumn{2}{|c|}{ PLACE OF PRODUCTION } & \multirow{2}{*}{$\begin{array}{c}\text { Kilos } \\
\text { per } \\
\text { Hectolitre }\end{array}$} & \multirow{2}{*}{$\begin{array}{c}\text { Lbs. } \\
\text { per } \\
\text { Bushel } \\
\text { (W' CIESTER) }\end{array}$} \\
\hline & & & & DISTRICT & PROVINCE & & \\
\hline $33 \pi$ & French & Wheat & Nogueras y Avalos & F. Madero & Buenos Aires & 83.200 & 64.6 \\
\hline 338 & ," & , & Santamarina é hijos & Pehuajó & . & 82.050 & 63.7 \\
\hline 339 &, & ," & Traverso Hnos & Puan &, & $\$ 2.300$ & 63.9 \\
\hline 340 & ," & ", & Chillado, Genta y Cia. & Bolivar &, & $\$ 1.150$ & $63 .-$ \\
\hline 341 & ," & , & S. Unzué é hijos & Las Martinetas &, & 81.050 & $63 .-$ \\
\hline 342 & ", & ," & Murature, Saenz y Cia. & Cazón & ," & 80.700 & 62.7 \\
\hline 343 & , & " & S. Unzué é hijos & Chivilcoy & ," & 80.450 & 62.5 \\
\hline 344 & ," & ", & Lucione y Ferrari & San Pedro & ," & 81.4 .00 & 63.2 \\
\hline 345 & ," & ," & Balleto $y$ Bidart & El Moro & ", & 80.700 & 62.7 \\
\hline 34.6 & " & " & C. Martinez & Chacabuco & ", & 81.250 & 63.1 \\
\hline $3+7$ & .. & " & S. Unzué ć hijos & Coronel Suarez & ", & 82.300 & 63.9 \\
\hline 348 & ,. & ". & Devoto, Balbiani y Cia. & La Zanja & , & 82.850 & 64.4 \\
\hline 349 & " & $\therefore$ & Juan Devoto (hijo) & C. Casares & , & 83.100 & 64.6 \\
\hline 350 & , & ," & Bayona y Terrazzini & Drysdale & , & 83.800 & 65.1 \\
\hline 351 &, & , & A. Marconi & Trenque Lauquen & ," & 79.750 & 62.- \\
\hline 352 & , & ," & Palacios Hnos. & F. Madero & , & 79.450 & 61.7 \\
\hline 353 & , & ", & G. Lértora & Olascoaga & ,. & 82.300 & 63.9 \\
\hline 354 & ," & $"$ & Verge é Iglesias. & Olavarria &, & $83 .-$ & 64.5 \\
\hline 355 & , & , & Viuda de Apellaniz & Gencral Chaves & ," & 81.250 & 63.1 \\
\hline 356 & $"$ & ," & Chillado, Genta y Cia. & Primera Junta & " & 82.550 & 64.1 \\
\hline 357 & $"$ & , & Devoto, Balbiani y Cia. & F. Madero & .. & 80.800 & 62.8 \\
\hline 358 & , & , & Muratore, Saenz y Cia. & General Lamadrid & ," & 80.550 & 62.6 \\
\hline 359 & $"$ & $"$ & $" \quad, \quad \quad$, & Mercedes & " & 82.400 & $64 .-$ \\
\hline 360 & $"$ & , & Calvo y Cobo & C. Casares &, & 83. - & 64.5 \\
\hline 361 &, & $"$ & José M. Mendez & Bonifacio & ," & 81.950 & 63.6 \\
\hline 362 & ", & , & César Galoppo & Castellanos & Santa Fé & 82.550 & 64.1 \\
\hline 363 & , &, & Seco Hnos. & Río $3^{\circ}$ A bajo & Córdoba & 82.4 .00 & $64 .-$ \\
\hline 364 & , &, & Juan Marriezcurrena & Genera1 Villegas & Buenos Aires & 80.900 & 62.9 \\
\hline 365 & ," & , & Raimundo Salazar \& Cia. & F. Madero & , & 79.750 & $62 .-$ \\
\hline 366 & ," & , & Antonio Biordo & Lincoln & , & 81.4 .00 & 63.2 \\
\hline 367 & , & ,. & S. Unzué é hijos & Pirovano & , & 80.100 & 62.2 \\
\hline 368 & ," &, & Palacios Hnos. & F. Madero & ," & $7 \pi .550$ & 60.2 \\
\hline 369 & ", & $"$ & Gandencio Rossi & Lujan & ", & 79.200 & 61.5 \\
\hline 370 & $"$ & ,. & Santiago Balbi y Cia. & Junin & ", & 81.950 & 63.6 \\
\hline 371 & ," & ," & Pedro Pautasso & Las Colonias & Santa Fé & 82.650 & $6 \div .2$ \\
\hline 372 & ," & $\because$ & Nicolás Panadero & Trenque Lauquen & Buenos Aires & 80.100 & 62.2 \\
\hline 373 & ", & , & Antonio Bello & Bragado & $"$ & 81.600 & 63.4 \\
\hline 374 & $"$ &. & Miguel Rizzi & 25 de Mayo &, & 80.450 & 62.5 \\
\hline 375 & - & $"$ & Pedro Estanguct & Lahonlaye & Córdoba & 80.450 & 62.5 \\
\hline 376 & ," & , & Palacios Hnos. & 9 de Julio & Buenos Aircs & 82.400 & $64 .-$ \\
\hline 377 & Russian V & Wheat & Devoto, Balbiani y Cia. & Guanaco & , & 83.350 & 64.7 \\
\hline 378 & $-\quad$, & ," & H. Higgins & Cambaceres & , & 83.900 & 65.2 \\
\hline
\end{tabular}




\begin{tabular}{|c|c|c|c|c|c|c|c|}
\hline \multirow{2}{*}{ No. } & \multirow{2}{*}{\multicolumn{2}{|c|}{ PRODUCT }} & \multirow{2}{*}{$E X H I B I T O R$} & \multicolumn{2}{|c|}{ PLACE OF PRODUCTION } & \multirow{2}{*}{$\begin{array}{c}\text { Kilos } \\
\text { per } \\
\text { Hectolitre }\end{array}$} & \multirow{2}{*}{$\begin{array}{c}\text { Lbs. } \\
\text { per } \\
\text { Bushel } \\
\text { (1)'CHE.TFP) }\end{array}$} \\
\hline & & & & DISTRICT & PROVINCE & & \\
\hline 379 & Russian WV & Theat & Devoto, Balbiani y Cia. & Prinera Junta & Buenos Aires & $84 . \quad-$ & 65.3 \\
\hline 380 & ," & ," & J. M. Mendez & Islas & ," & 85.050 & 66.1 \\
\hline 381 & , &, & P. y A. Lanusse & Lineoln & ", & 83.900 & 65.2 \\
\hline 382 & , & ," & Deroto, Balbiani y Cia. & Dussaud & , & 84.500 & 65.6 \\
\hline 383 & , & ," & Clillado, Genta y Cia. & Primera Junta & ," & 83.700 & $65 .-$ \\
\hline 384 & ", & , & Viuda de $\Lambda$ pellaniz & Neeochea & ," & 83.800 & 65.1 \\
\hline 385 &, & ," & , , , & Bonifaeio & ", & $83 .-$ & 64.5 \\
\hline 386 &, & ", & J. Mariezeurrena & General Villegas & ", & 83.100 & 64.6 \\
\hline 387 & ," & ," & J. Neumon & Carlité & ", & 83.350 & 64.7 \\
\hline 358 & , & ", & Pcllegrino Rossi & $\Lambda z u 1$ & ," & $\$ 3.200$ & 64.6 \\
\hline 389 & ", & , & Juan Kctta & Coronel Suarez & ," & 85.400 & 66.3 \\
\hline 390 & ", & ," & Rascougnet Hnos. & Tandil & " & 80.450 & 62.5 \\
\hline 391 & , & , & Raimundo Salazar y Cia. & F. Madcro & ", & 82.650 & 64.2 \\
\hline 392 & ," & " & Tiburcio Rineon & Coronel Suarez & ", & 83. - - & 64.5 \\
\hline 393 & ", & , & Juan B. Pinaquy & Laprida & ", & 80.550 & 62.6 \\
\hline 394 & ," & ", & José Gonzalez & Trenque Lauquen & ", & 82.400 & $64 .-$ \\
\hline 395 & " & ," & Juan A. Sanchez & Coronel Pringles & ", & 84.700 & 65.8 \\
\hline 396 & ", & ," & Pcdro Sandungaray & Bahia Blanea & ", & 81.150 & 63.- \\
\hline 397 & ", & ", & D. Foureade & Unión & Córdoba & 82.400 & $64 .-$ \\
\hline 398 & ., & , & Pedro Lopez & Gencral Dorrego & Buenos $\Lambda$ ires & 82.550 & 64.1 \\
\hline 399 & " & ," & Agustin Eloriaga & A. Alsina & ", & 81.950 & 63.6 \\
\hline 400 & ," & , & Domingo E. Rodriguez & Tres Arroyos & ," & 82.850 & 64.4 \\
\hline 4.01 & $"$ & ", & D. Fourcade & Unión & Córdoba & 84.700 & 65.8 \\
\hline 402 & " & , & José Coloredo & Trenque Latuquen & Buenos Aires & 82.550 & 64.1 \\
\hline 4.03 & $"$ & , & Amadeo de la Cuadra & Coronel Pringles & , & S6.550 & $6 \pi .2$ \\
\hline 4.04 & Piedmontesc & Wheat & P. y A. Lanusse & Bandcraló & , & 84.250 & 65.5 \\
\hline 4.05 & " & ", & Ballcto y Bidart & Chiclana & ", & $84 .-$ & 65.3 \\
\hline 406 & ," & ", & Ojea y García & Pehuajó & ", & 84. - & 65.3 \\
\hline 4.07 & " &, & L. Dreyfus & Passo & ", & 85.300 & 66.3 \\
\hline 408 & ," &, & Nogueras y Avalos & La Flora & ", & 84.600 & 65.7 \\
\hline 409 & ", & ," & Balleto y Bidart & Chielana & ", & $\$ 4.950$ & $66 .-$ \\
\hline 410 & " & ," & A. Labro & Chivilcoy & ", & 83.100 & 64.6 \\
\hline 411 & ", & , & Pedro Foos & San Justo & Córdoba & 80.800 & 62.8 \\
\hline 412 & ," &, & Tettamanti Hnos. & San Pedro & Buenos Aires & 79.200 & 61.5 \\
\hline 413 & ," & ", & Raimuudo Salazar y Cía. & F. Madero & ", & 83.900 & $6 \overline{5} .2$ \\
\hline 414 & Italian $W h$ & heat & Batutista Oherto & Iriondo & Santa Fé & 84.600 & 65.7 \\
\hline 415 & ," & ", & Simón Colombo & Diamante & Entrc Ríos & 83.700 & 65.- \\
\hline 416 & $"$ & , & Llomurini y Cía. & Quilmes & Buenos Aires & 82.200 & 63.8 \\
\hline 417 & ", & " & David Baroni & San Justo & Santa Fé & 83. - - & 64.5 \\
\hline 418 & " & ", & Bernardo Villarino & Nogoya & Entre Ríos & 83.100 & 64.6 \\
\hline 4.19 & , & , & Josć Catac & Paraná & , & 81.850 & 63.6 \\
\hline $4 \div 0$ & Lombardy W & Vheat & A. Marconi & A. del Valle & Euenos Aires & $82 .-$ & 63.7 \\
\hline
\end{tabular}




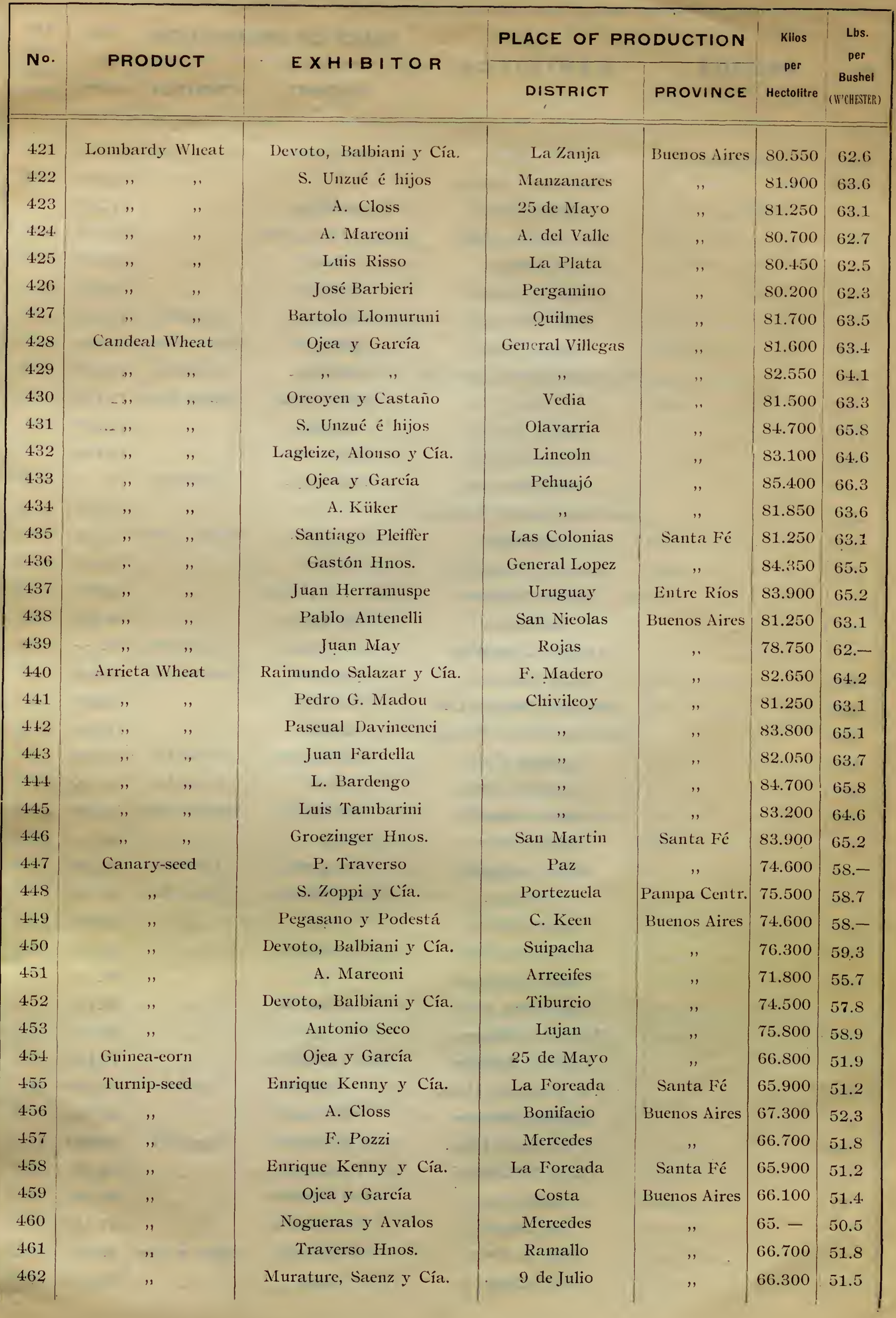




\begin{tabular}{|c|c|c|c|c|c|c|}
\hline \multirow{2}{*}{ No. } & \multirow{2}{*}{ PRODUCT } & \multirow{2}{*}{ EXHIBITOR } & \multicolumn{2}{|c|}{ PLACE OF PRODUCTION } & \multirow{2}{*}{$\begin{array}{c}\text { Kilos } \\
\text { per } \\
\text { Hectolitre }\end{array}$} & \multirow{2}{*}{$\begin{array}{c}\text { Lbs. } \\
\text { per } \\
\text { Bushel } \\
\text { (II'chlesterk) }\end{array}$} \\
\hline & & & DISTRICT & PROVINCE & & \\
\hline 463 & Turnip-seed & Porta Hnos. & San Pedro & Buenos Aires & 66.900 & 52.- \\
\hline 464 & , & Baldomero Llerena & $"$ & ," & 66.200 & 51.4 \\
\hline 465 & Linseed & Traverso Hnos. & Guticrrez & ," & 71.200 & 55.3 \\
\hline 466 & , & Juan Devoto (hijo) & General Sarmiento & $"$ & 72.500 & 56.3 \\
\hline 467 & , & A. Delfino & Toro & $"$ & 73.100 & 56.8 \\
\hline 468 & , & Ojea y García & Mercedes & $"$ & 69.500 & 54.- \\
\hline 469 & $"$ & Pedro Estanguet & Laboulaye & Córdoba & 72.300 & 56.2 \\
\hline 4.70 &, & Ojea y Gareía & Mereedes & Buenos Aires & 69.900 & 54.3 \\
\hline 4.71 & ," & Salaberry, Lalor y Berectche & Laboulaye & Córdoba & 71.800 & 55.7 \\
\hline 472 &, & A. Velaz y Ciál. & Manzanares & Buenos Aires & 71.700 & 55.6 \\
\hline 473 & , & Ojca y García & Mercedes & ", & 71.500 & 55.5 \\
\hline 474 & , & S. Unzué é hijos & Chacabueo & $"$ & 71.100 & 55.2 \\
\hline 475 & , & Pedro Estanguet & Laboulaye & Córdoba & $72 .-$ & 55.9 \\
\hline 476 & ", & Salaberry, Lalor y Bereetehe & ", & $"$ & 71.900 & 55.8 \\
\hline 477 & ", & Eloy Velaz & San Pedro & Buenos Aires & 70.400 & $5+.7$ \\
\hline 4.78 & , & Silvestre Calandrelli & Lujan & $"$ & 68.400 & 53.1 \\
\hline 479 & , & Mauro Maderua & Exalt. de la Cruz & $"$ & 69.900 & 54.3 \\
\hline 480 & $"$ & José Alacia & San Pedro &, & 69.300 & 53.8 \\
\hline 4.81 & , & Antonio Caramelino & M. Juarez & Córdoba & $69 .-$ & 53.6 \\
\hline 482 & ", & P. V. Jauregui & Jauregui & Buenos Aires & 68.600 & 53.3 \\
\hline 483 & $"$ & Murature, Saenzy Cía. & Suipacha & ," & 70.600 & 54.8 \\
\hline 484 & $"$ & Salaberry, Lalor y Bercetche & Merlo & ," & 71.300 & 55.4 \\
\hline 485 & , & Ayrando Hnos. & Castellanos & Santa Fé & 69.300 & 53.8 \\
\hline 4.86 & , & Juan Pickenstain & Río $4^{\circ}$ & Córdoba & 69.800 & 54.2 \\
\hline 487 & , & Gaspar Selıonith & San Pedro & Buenos Aires & 72.700 & 56.4 \\
\hline 4.88 & , & Porta Hnos. & $"$ & , & 66. - & 51.3 \\
\hline 489 &, & Felipe Carignano & San Justo & Córdoba & 69.500 & $54 .-$ \\
\hline 490 & , & C. Galope & Las Colonias & Santa Fé & 69.400 & 53.9 \\
\hline 491 & , & Bunge y Born &.$\quad$, & , & 67.200 & 52.2 \\
\hline 492 & , & Carlos Merchen & Castellanos & $"$ & 68.850 & 53.5 \\
\hline 493 & , & Pedro Guglielmone & $"$ & ," & 69. - & 53.6 \\
\hline 494 & , & Berganiasco Hnos. & Las Colonias & $"$ & 70.100 & 54.4 \\
\hline 495 & , & Marta de Hungenet & 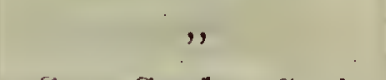 & " & 69.100 & 53.6 \\
\hline 496 &, & Augnsto Tsehopp & San Carlos Sud & $"$ & 71.100 & 55.6 \\
\hline 497 & , & J. S. Baumann Hnos. & Union & Córdoba & 69.500 & 54.- \\
\hline 498 & , & José Guglielmone & San Justo & $"$ & 69.200 & 53.7 \\
\hline 499 & ", & D. Pirovano Hinos. & Castellanos & Santa Fé & 68.900 & 53.5 \\
\hline 500 & $"$ & Juan Bolaniperti & La Capital & , & 69.700 & 54.1 \\
\hline 501 & , & Isidro Vega & Las Colonias & , & 69.600 & 54.1 \\
\hline 502 & , & Luis Capeletti & Reeonquista & " & 67.150 & 52.2 \\
\hline 503 & , & Juan B. Arana & - Pergamino & Buenos Aires & 69.900 & 54.3 \\
\hline 504 & $"$ & B. Casey & Lujan & , & $\tau 0.600$ & 54.8 \\
\hline
\end{tabular}




\begin{tabular}{|c|c|c|c|c|c|c|}
\hline \multirow{2}{*}{ No. } & \multirow{2}{*}{ PRODUCT } & \multirow{2}{*}{$E X H I B I T O R$} & \multicolumn{2}{|c|}{ PLACE OF PRODUCTION } & \multirow{2}{*}{$\begin{array}{c}\text { Kllos } \\
\text { per } \\
\text { Hectolitre }\end{array}$} & \multirow{2}{*}{$\begin{array}{c}\text { Lbs. } \\
\text { per } \\
\text { Büshel } \\
\text { (W'CHESTER) }\end{array}$} \\
\hline & & & DISTRICT & PROVINCE & & \\
\hline 505 & Linsecd & Martin Badollotti & San Lorenzo & Santa Fé & 67.900 & 52.8 \\
\hline 506 & ," & Juan Iturbe & Unión & Córdoba & 67.900 & 52.8 \\
\hline 507 & ," & G. Irizar Hnos. & Pergamino & Buenos Aires & 70.800 & $55 .-$ \\
\hline 508 & $"$ & Bartolo Schunk & Diamante & Entre Ríos & 70.200 & 54.5 \\
\hline 509 & ", & P. y N. Antuña & Las Colonias & Santa Fé & 69.300 & 53.8 \\
\hline 510 & $"$ & Giroldi y Nano & Constitueión & , & 67.800 & 52.7 \\
\hline 511 & $"$ & Francisco Saforcada & Junin & Bucnos Aires & 67.200 & 52.2 \\
\hline 512 & $"$ & Luis Cabrini & Iriondo & Santa Fé & 69.300 & 53.8 \\
\hline 513 &, & José Bonetti & Bahía Blanca & Buenos Aires & 68.500 & 53.2 \\
\hline 514 & ," & Pablo Pierini & Castellanos & Santa Fé & 69.800 & 54.2 \\
\hline 515 & ," & Pedro Godino & $"$ &, & 69.800 & 54.2 \\
\hline 516 & , & Felix Lazarino & General Lopez & , & 69.400 & 53.9 \\
\hline 517 & ", & Miguel Toceo & Iriondo & , & 69.200 & 53.7 \\
\hline 518 & ", & Leopoldo Gezzi & Castellanos &, & 69.600 & 54.1 \\
\hline 519 & $"$ & Miguel Barres & General Belgrano & Buenos Aires & 69.700 & 54.1 \\
\hline 520 & , & Braida Hnos. & San Cristobal & Santa Fé & 67.600 & 52.5 \\
\hline 521 & ,. & Lorenzon Giaeosa & San Gerónimo & , & 67.800 & 52.7 \\
\hline 522 &, & I. y s. Griffa & Castellanos & , & 70.100 & 54.1 \\
\hline 523 & ", & Antonio Felcaro & San Gerónimo & , & 71.200 & 55.3 \\
\hline 524 & ." & Isaac Babich & Villaguay & Eintre Ríos & 71.200 & 55.3 \\
\hline 525 & ," & Depetres Hnos. & Gencral Pinto & Buenos Aires & 69.300 & 53.8 \\
\hline 526 & ," & Salvador Busso & Lineoln & ," & 70.400 & 54.7 \\
\hline 527 & ", & Alejandro Labourcaux & Lujan &, & 70.500 & 54.8 \\
\hline 528 & ", & Luis Rossi & Colón & ", & 69.800 & 54.2 \\
\hline 529 & ," & Enrique Charles & San Carlos Sud & Santa Fé & 69.200 & 53.7 \\
\hline 530 &, & P. S. Maggi & Lincoln & Buenos Aires & $69 .-$ & 53.6 \\
\hline 531 & , & Antonio Gorospe & San Nieolás & $"$ & 70.500 & 54.8 \\
\hline 532 & ,. & Andres Celctti & Giles & ," & 70.800 & 55. \\
\hline 533 &, & Juan Brusa & Río Segundo & Córdoba & 69.500 & $54 .-$ \\
\hline 534 & " & Augusto Sixte & Puan & Buenos Aires & 68. - & 52.9 \\
\hline 535 & " & E. é J. Fabre & Las Colonias & Santa Iré & 69.300 & 53.8 \\
\hline 536 & $"$ & Albino E. Reffino & Paraná & Entre Ríos & 68.800 & 53.5 \\
\hline 537 & ," & Eduardo Weibes & San Cristobal & Santa Fé & 67.300 & 52.2 \\
\hline 538 & , & Angel Godio & Las Colonias & ", & $68 .-$ & 52.9 \\
\hline 539 & ," & Domingo Mondino & San Martin & , & 70.500 & 54.8 \\
\hline 540 & ", & Pasquale Olivo y Cía. & Castellanos & ", & 70.200 & 54.5 \\
\hline 541 & ," & S. Raeca Hnos. & Río $3^{\circ}$ Arriba & Córdoba & 67.400 & 52.3 \\
\hline 542 & , & Lorenzo Ralero & Diamante & Entre Ríos & 68.600 & 53.3 \\
\hline $5+3$ & ," & José Sehmit & Castellanos & Santa Fé & $69 .-$ & 53.6 \\
\hline $5+4$ & ", & Miguel Primo & Nogoyá & Entre Ríos & 68. -- & 52.9 \\
\hline 545 & $"$ & Pedro Estanguet & Laboulaye & Cordoba & 67.100 & 52.2 \\
\hline 54.6 & $"$ & Juan Sieber & San Gerónimo & Santa Fé & 70.500 & 54.8 \\
\hline
\end{tabular}




\begin{tabular}{|c|c|c|c|c|c|c|}
\hline \multirow{2}{*}{ No. } & \multirow{2}{*}{ PRODUCT } & \multirow{2}{*}{ EXHIBITOR } & \multicolumn{2}{|c|}{ PLACE OF PRODUCTION } & \multirow{2}{*}{$\begin{array}{c}\text { Kilos } \\
\text { per } \\
\text { Hectolitre }\end{array}$} & \multirow{2}{*}{$\begin{array}{c}\text { Lbs. } \\
\text { per } \\
\text { Bushel } \\
\text { (W'CIESTER) }\end{array}$} \\
\hline & & & DISTRICT & PROVINCE & & \\
\hline $54 . \overline{7}$ & Linseed & Juan Balari & Las Colonias & Santa Fé & 67.700 & 52.6 \\
\hline 54.8 &, & H. Duqui & San Pedro & Bucnos Aircs & 70.200 & 54.5 \\
\hline 549 & , & Luis Mortinelli & Río Cuarto & Córdoba & 69.200 & 53.7 \\
\hline 550 & ,. & Bautista Dovis & San Justo & Santa Fé & $69 .-$ & 53.6 \\
\hline 551 & ," & Miguel Mahon & Constitución & " & 71.300 & 55.4 \\
\hline 552 & , & Gustavo Helmbold & Las Colonias & " & 69.600 & 54.1 \\
\hline 553 & ," & José Monteverde & San Pedro & Bucnos Aires & 70.600 & 54.8 \\
\hline 554 & , & Josẹ́ Franticelli & Concordia & Entre Ríos & 68. - & 52.9 \\
\hline $55 \overline{5}$ & ," & Vicente Madio & Iriondo & Santa Fé & 70.200 & 54.5 \\
\hline 556 &, & Traverso Hnos. & Constitución & , & 69.300 & 53.8 \\
\hline 557 & , & Olivera Hnos. & Castellanos & , & 68.800 & 53.5 \\
\hline 558 &, & Carlos Motta & Colon & Buenos Aires & 69.300 & 53.8 \\
\hline 559 & , & Antonio Marensi & General Lopez & Santa Fé & 67.600 & 52.5 \\
\hline 560 & , & Roque Velazquez & Nogoya & Entrc Ríos & 69.800 & 54.2 \\
\hline 561 & ," & Guillermo Tonelli & General Pinto & Buenos Aires & 69.900 & 54.3 \\
\hline 562 & ," & Fernando Colombo & San Cárlos Norte & Santa Fé & 68.800 & 53.5 \\
\hline 563 & $"$ & Francisco Estrada & S. Antonio de Areco & Buenos Aires & 71.900 & 55.8 \\
\hline 564 & $"$ & S. Mandrin & San Cristobal & Santa Fé & 69.300 & 53.8 \\
\hline 565 & ," & Ramón Hernandez & San Nicolás & Bucnos Aircs & 67.300 & 52.2 \\
\hline 566 & , & Santiago Picassi & San Martin & Santa Fé & $69 .-$ & 53.6 \\
\hline 567 &, & Fondevila y Español & San Gerónimo & , & 70.900 & $55 .-$ \\
\hline 568 & , & Felipe Dreizigacker & Paraná & Entre Ríos & 67.250 & 52.2 \\
\hline 569 & , & Antonio Fabris & San Justo & Santa Fé & 69.500 & 54.- \\
\hline 570 & , & Enrique Nicola & Las Colonias & , & 68.750 & 53.4 \\
\hline 571 & " & Franeisco Palá & Chacabuco & Buenos Aires & 67.300 & 52.2 \\
\hline 572 & $"$ & Ignacio Guraya & Carcarañá & Santa Fé & $70 .-$ & 54.3 \\
\hline 573 & , & Felix Gábctto & San Martin & $"$ & 69.800 & 54.2 \\
\hline 574 & , & Francisco Mehaca & San Lorenzo & , & 67. & 52.1 \\
\hline 575 & $"$ & Patricio B. Dillon & San Andrés de Giles & Buenos Aires & $70.800 \mid$ & 55.- \\
\hline 576 & , & Pedro Gordolich & Rosario & Santa Fé & 69.800 & 54.2 \\
\hline 577 & , & Bernardo Barbera & Iriondo & , & $70 .-$ & 54.3 \\
\hline 578 & , & Sucesión de Josć Canesa & General Lopez & , & 67.500 & 52.4 \\
\hline 579 & , & Parada y Alvarez & Chacabuco & Buenos Aires & $70 .-$ & 54.3 \\
\hline 580 & $"$ & Roberto Cima & Colon &, & 68.300 & 53.- \\
\hline 581 & ", & Bernardo Piana & Castellanos & Santa Fé & 67.900 & 52.8 \\
\hline 582 & , & Fcderico Grün & Paraná & Entre Ríos & 71. & 55.1 \\
\hline 583 & , & Antonio Lopez & Carmen de Areco & Buenos Aires & $71 .-$ & 55.1 \\
\hline 584 & $"$ & Antonio Chixmi & San Justo & Santa Fé & 67.600 & 52.5 \\
\hline 585 & $"$ & Bautista Pighin & , & , & 69.100 & 53.6 \\
\hline 586 & , & Bonaluza Hnos. & Bragado & Buenos Aires & 70.500 & 54.8 \\
\hline 587 & ", & Digiorgio Hnos. & Estción de la Cruz & " & 71.900 & 55.8 \\
\hline 588 & $"$ & Bassi Hnos. & Las Colonias & Santa Fé & 69. & 53.6 \\
\hline
\end{tabular}




\begin{tabular}{|c|c|c|c|c|c|c|c|}
\hline \multirow{2}{*}{ No. } & \multirow{2}{*}{\multicolumn{2}{|c|}{ PRODUCT }} & \multirow{2}{*}{ EXHIBITOR } & \multicolumn{2}{|c|}{ PLACE OF PRODUCTION } & \multirow{2}{*}{$\begin{array}{c}\text { Kilos } \\
\text { per } \\
\text { Hectolitre }\end{array}$} & \multirow{2}{*}{$\begin{array}{c}\text { Lbs. } \\
\text { per } \\
\text { Bushel } \\
\text { (W'CHESTER) }\end{array}$} \\
\hline & & & & DISTRICT & PROVINCE & & \\
\hline 589 & Linsec & & Cristobal Zati & Paraná & Entre Ríos & $67 .-$ & 52.1 \\
\hline 590 & , & & Emilio N. Mora & 25 de Mayo & Bucnos Aires & 70.100 & 54.4 \\
\hline 591 & , & & Viuda de Ovalle Hnos. & San Martin & Santa Fé & 69.800 & 54.2 \\
\hline 592 & , & & Rizzieri y Marini Hnos. & Reconquista & " & 69.200 & 53.7 \\
\hline 593 & ," & & A. Vallasi y Cía. & Gral. Lopez. & $"$ & 70.700 & $55 .-$ \\
\hline 594 & " & & Vieente Allone & 25 de Mayo & Buenos Aires & $69 .-$ & 53.5 \\
\hline 595 & ," & & Pedro Bassi & Uringuy & Entre Ríos & 71. - & 55.1 \\
\hline 596 & ," & & Boretto Hnos. & Castcllanos & Santa Fé & 70.300 & 54.6 \\
\hline 597 & ," & & Juan I.udi & Paraná & Entre Ríos & 69.800 & 54.2 \\
\hline 598 & , & & Domingo Fourcade & Unión & Cordoba & 68.200 & 52.9 \\
\hline 599 &, & & Lorenzo Negro & San Martin & Santa Fé & 68. - & 52.9 \\
\hline 600 & ", & & Martin Otamendi & Victoria & Entre Ríos & $67 .-$ & 52.1 \\
\hline 601 & " & & Andres Calletti & Giles & Buenos Aires & 70.700 & $55 .-$ \\
\hline 602 &, & & Bartolo Dalmaso & San Martin & Santa Fé & $66 .-$ & 51.3 \\
\hline 603 & ," & & Ramón Martinez & General Villegas & Buenos Aires & 69.250 & 53.8 \\
\hline 604 & ," & & Eugenio Pidesti & Rosario & Santa Fé & 69.100 & 53.7 \\
\hline 605 & ," & & Justiniano Adalo & ," & , & 68.200 & 52.9 \\
\hline 606 &, & & Olacehea y Aspiri & Gral. Lopez & , & 69.200 & 53.7 \\
\hline 607 & Barletta I & Wheat & J. W. de Kober & Paraná & Entre Ríos & 81.250 & 63.1 \\
\hline 608 & $"$ & ," & Ramón Martinez & General Villegas & Buenos Aires & 85.400 & 66.3 \\
\hline 609 & ", & , & Roberto Ciema & Colon & , & 84.150 & 65.4 \\
\hline 610 &, & ," & Vietoriano Videau & Necoehea & , & 83.700 & $65 .-$ \\
\hline 611 & , & , & José Canero & Iriondo & Santa Fé & 84. - & 65.3 \\
\hline 612 & " & ," & Joaquin Aduniz & Pringles & Buenos Aires & 85.500 & 66.4 \\
\hline 613 & ", & , & Enrique Charles & Las Colonias & Santa Fé & 82.850 & 64.4 \\
\hline 614 & ", & ", & F. Castea Madariaga & Tandil & Buenos Aires & 81.500 & 63.3 \\
\hline 615 & ," & ," & A. Panasi y Cía. & Gral. Lopez & Santa Fé & 83.550 & 64.9 \\
\hline 616 & , & ," & Diego Benvenuto Hnos. y Cía. & M. Juarez & Córdoba & 85.650 & 66.5 \\
\hline 617 & ." & ,. & Juan Litre & Saavedra & Bucnos Aires & 85.150 & 66.2 \\
\hline 618 & " & .. & Viecnte Albeverio & Giles & , & 83. - & 64.5 \\
\hline 619 & ," & ," & Jorge Andant & Guaminí & ", & 83.800 & 65.1 \\
\hline 620 & , & ", & Carlos M. Casares & Bolivar & ", & 85.400 & 66.3 \\
\hline 621 & ," & " & Bosch y Tarrau & San Gerónimo & Santa Fé & 82.650 & 64.2 \\
\hline 622 & " & ", & José Scagliotti & Belgrano & $"$ & 85.850 & 66.7 \\
\hline 623 & ," & ," & Gorosito y Molquide & Juarez & Buenos Aires & 83.700 & 65.- \\
\hline 624 & , & ." & Atilio Carra & Río $3^{\circ}$ abajo & Córdoba & 84.950 & 66.- \\
\hline 625 & , & ," & Luis Lauguet & General Pinto & Buenos Aires & 84.850 & 65.9 \\
\hline 626 & , & , & Juan Caletti & Río $3^{\circ}$ abajo & Córdoba & 83.900 & 65.2 \\
\hline 627 & ," & ., & Juan M. Seró & Uruguay & Entre Ríos & 81.600 & 63.4 \\
\hline 628 & ," & ," & Vietorio Di Lorenzi & San Martin & Santa Fé & 80.350 & 62.4 \\
\hline 629 & ", & ,f & Antonio Marelli & San Jisto & $"$ & 83.350 & 64.7 \\
\hline 630 & $"$ & , & Luis Campi & Diamante & Entre Ríos & 83.350 & 64.7 \\
\hline
\end{tabular}




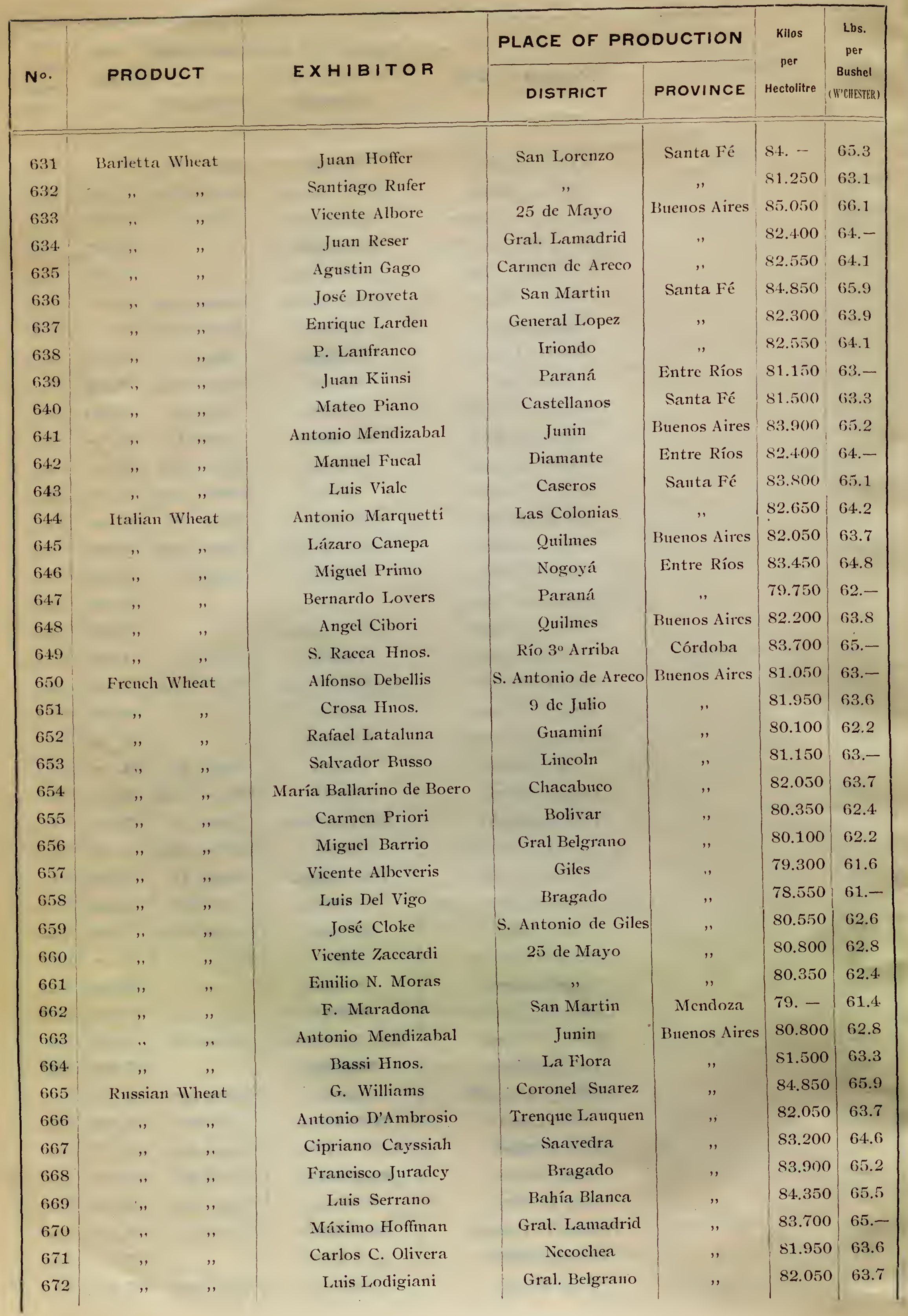




\begin{tabular}{|c|c|c|c|c|c|c|c|}
\hline \multirow{2}{*}{ No. } & \multirow{2}{*}{\multicolumn{2}{|c|}{ PRODUCT }} & \multirow{2}{*}{ EXHIBITOR } & \multicolumn{2}{|c|}{ PLACE OF PRODUCTION } & \multirow{2}{*}{$\begin{array}{c}\text { Kilos } \\
\text { per } \\
\text { Hectolitre }\end{array}$} & \multirow{2}{*}{$\begin{array}{c}\text { Lbs. } \\
\text { per } \\
\text { Bushel } \\
\text { (W'CHESTER) }\end{array}$} \\
\hline & & & & DISTRICT & PROVINCE & & \\
\hline 1 & Barletta & Wheat & E. Eckell & Halsey & Buenos Aires & 85.200 & 66.2 \\
\hline 2 & $"$ & , & Leon Lascaray & Laboulaye & Cordoba & 84.700 & 65.8 \\
\hline 3 & , &, & Pegasano y Podestá & Bayauca & Buenos Aires & 83.400 & 64.7 \\
\hline 4. & ," &, & Lcon Lascaray & Laboulaye & Cordoba & 84.600 & 65.7 \\
\hline 5 &, & ," & Escudero Hnos. & D. de Alvear & , & 83.200 & 64.6 \\
\hline 6 &, & ,. & B. Bernhard & , & , & 82.400 & $64 .-$ \\
\hline 7 &, & , & $"$ &, &, & 84.350 & 65.5 \\
\hline 8 & , & ," & " &, &, & 82. - & 63.6 \\
\hline 9 &, & , & Escudero Hnos. & Maggiolo &, & $\$ 2.550$ & 64.1 \\
\hline 10 & ." & ," & Palacios Hnos. & Alberti & Buenos Aires & 83.100 & 64.6 \\
\hline 11 & " & , & Salaberry, Lalor y Bercetche & Laboulaye & Cordoba & 81.850 & 63.6 \\
\hline 12 & . & ," & A. Bonorino & General Pinto & Buenos Aires & 84.100 & 65.4 \\
\hline 13 & ," & , & Pegasano y Podestá & Bayauca & , & 83.700 & 65.- \\
\hline 14 & , & ," & & Bolivar &, & 83.800 & 65.1 \\
\hline 15 & $"$ & $"$ & Verge é Iglesias & San Patricio &, & 85.150 & 66.2 \\
\hline 16 &, &, & Devoto, Balbiani y Cia. & Monte & , & 86.5 .50 & 67.2 \\
\hline 17 & $"$ & $"$ & R. de la Riestra & General Pinto & , & 83.450 & 64.8 \\
\hline 18 &, & ," & J. M. Mendez & Carhué & , & 81.300 & 63.1 \\
\hline 19 &, & ", & B. Bernhard & Alvear & Cordoba & 84.150 & 65.4 \\
\hline 20 & , & $"$ & A. Marconi & Trenque Lauquen & Buenos Aires & 85.900 & 66.7 \\
\hline 21 & , & , & Escudero Hnos. & General Villegas & , & 84.500 & 65.6 \\
\hline 22 &, & , & A. Bonorino & Pirovano & , & 83.200 & 64.6 \\
\hline 23 & ," & , & Lucione y Ferrari & General Sarmiento & , & 80.300 & 62.4 \\
\hline 24 &, & ," & Ayarragaray y Gowland & Gowland & ," & 86.500 & 67.2 \\
\hline 25 & ", &, & E. Eckell & Halsey & $"$ & 84.600 & 65.7 \\
\hline 26 & $"$ & ", & J. M. Mendez & Pehuajó & , & 84.350 & 65.5 \\
\hline 27 &, &. & Escudero Hnos. & 25 de Mayo &, & 83.700 & $65 .-$ \\
\hline 28 & ," & ," & B. Bernhard & Alvear & Cordoba & 81.750 & 63.5 \\
\hline 29 & , , & " & A. Bonorino & General Pinto & Buenos Aires & $85 .-$ & $66 .-$ \\
\hline 30 & ", & $"$ & J. M. Mendez & Bonifacio & $"$ & 83.450 & 64.8 \\
\hline 31 & ," & ", & Escudero Hnos. & General Villegas &, & 84.300 & 65.5 \\
\hline 32 & ," & " & L. Dreyfus & Passo & ", & 84.350 & 65.6 \\
\hline 33 & ," &, & J. R. Ramsay & General Villegas & , & 82.850 & 64.4 \\
\hline 34 &, &, & Palacios Hnos. & Saladillo & , & $84 .-$ & 65.3 \\
\hline 35 & ," & , & Esscudero Hnos. & Lincoln & , & 85.050 & 66.1 \\
\hline 36 & ", & ", & A. Velaz y Cia. & Costa & ," & 86.550 & 67.2 \\
\hline 37 & $"$ & , & Ojea y García & 9 de Julio & ., & 83.700 & $65 .-$ \\
\hline 38 & ", &, & $", \quad$, & Pehuajó & ", & 82.200 & 63.8 \\
\hline 39 &, & ", & Ayarragaray y Gowland & Gowland & , & $86 .-$ & 66.8 \\
\hline 40 & , & ," & Pegasano y Podestá & Bayauca &, & 84.250 & 65.5 \\
\hline 41 & ", & ", & Genoud, Benvenuto, Martelli y Cia. & Pilar & , & 84.350 & 65.5 \\
\hline 42 & ," & ," & T. B. Arzeno & General Villegas & $"$ & 86.100 & 66.9 \\
\hline
\end{tabular}




\begin{tabular}{|c|c|c|c|c|c|c|c|}
\hline \multirow{2}{*}{ No. } & \multirow{2}{*}{\multicolumn{2}{|c|}{ PRODUCT }} & \multirow{2}{*}{$E X H|B| T O R$} & \multicolumn{2}{|c|}{ PLACE OF PRODUCTION } & \multirow{2}{*}{$\begin{array}{c}\text { Kilos } \\
\text { per } \\
\text { Hectolitre }\end{array}$} & \multirow{2}{*}{$\begin{array}{c}\text { Lus. } \\
\text { per } \\
\text { Bushel } \\
\text { (W'Cl ESTER) }\end{array}$} \\
\hline & & & & DISTRICT & PROVINCE & & \\
\hline 43 & Barletta $V$ & Wheat & Urquiza Hnos. & Los Toldes & Buenos Aires & 85.300 & 66.3 \\
\hline 44. & " & " & Santamarina é hijos & Pchuajó & , & 86.300 & $67 .-$ \\
\hline 45 & , & , & T. Marull & Las Flores & ,. & 83.800 & 65.1 \\
\hline 46 & , & ," & J. M. Mendez y Cia. & Pelutuais & , & 85. - & $66 .-$ \\
\hline 4.7 &, & , & H. Il uggines & Cambaceres & ", & 83.800 & 65.1 \\
\hline 48 & , & , & S. Unzuć é hijos & Carlos Tejedor & , & 84.500 & 65.6 \\
\hline 49 & ., & ," & ", & Costa & , & 85.300 & 66.3 \\
\hline 50 & , & ", & T. E. Bcll & Halscy & , & 86.650 & 67.3 \\
\hline 51 & , & , & P. Etchart & General Sarmiento & ", & 82.400 & $64 .-$ \\
\hline 52 &, & , & Ayarragaray y Gowland & Lincoln & , & 84.950 & 66.- \\
\hline 53 & , & , & T. E. Bell & Halsey & ", & $87 .-$ & 67.6 \\
\hline 54 & ", & ", & Pegasano y Podestá & Bayanca & ," & 83.100 & 64.6 \\
\hline 55 & , & , & A. Marconi & Trenque Lauquen & " & 86. - & 66.8 \\
\hline 56 & , & , & P. Etchart & Gencral Sarmicnto & ," & 82.550 & 64.1 \\
\hline 57 & , & , & H. Heiggines & Cambaceres & ". & 82.650 & 64.2 \\
\hline 58 & ", & , & Escudero Hnos. & Monte & , & 83.450 & 64.8 \\
\hline 59 & , & ," & Pegasano y Podestá & Larronde & Pampa Centr. & $87 .-$ & 67.6 \\
\hline 60 & ", & , & A. Velaz y Cia. & La Flora & Buenos Aires & 87.200 & 67.8 \\
\hline 61 & ", & ," & B. Bcrnhard & D. de Alrear & Córdoha & 84.600 & 65.7 \\
\hline 62 & , & , & Orcoyen, Castaños y Cia. & Alem & Buenos Aires & 86.350 & $67 .-$ \\
\hline 63 & , & , & Leon Lascaray & Laboulaye & Córdoba & $85 .-$ & 66.- \\
\hline 64 & ", & " & Escudero Hnos. & D. de Alvcar & " & 83.900 & 65.2 \\
\hline 65 & " & ," & Pcgasano y Porlestá & Larronde & Pampa Centr. & 86.800 & 67.4 \\
\hline 66 & $"$ & , & Salaberry, Lalor y Bercetehe & Guardia Vieja & Córdoba & 86.300 & 67.- \\
\hline 67 & , & ", & ," & $"$ & , & 85.600 & 66.5 \\
\hline 68 & , &, & A. Küiker & Rufino & ", & 84.150 & 65.4 \\
\hline 69 & , & , & Salaberry, Lalor y Bercetche & Guardia Vicja & ", & 82.200 & 63.8 \\
\hline 70 & ", & , & Santamarina é liijos & Lincoln & Buenos Aires & 85.650 & 66.5 \\
\hline 71 & ," & , & A. Bonorino & General Villegas & ", & 83.450 & 64.8 \\
\hline 72 & , & , & Vcrge é Iglesias & Berraondo & Pampa Centr. & 85.150 & 60.2 \\
\hline 73 & , & , & A. Velaz y Cia. & La Flora & Buenos Aires & 86.100 & 66.9 \\
\hline 74 & , & " & ", & San Mauricio & " & 86.650 & 67.3 \\
\hline 75 & , & , & A. Mollard & Guanaco & ", & 84.250 & 65.5 \\
\hline 76 & , & , & A. Marconi & Capilla & ", & 84.150 & 65.4 \\
\hline 77 & , & , & M. Fonda & Monte & ," & 84.850 & 65.9 \\
\hline 78 & ", & ", & A. Bonorino & Gencral Villegas & " & 83.900 & 65.2 \\
\hline 79 & , & , & S. Unzué é hijos & Concsa & ", & 85.150 & 66.2 \\
\hline 80 & , & , & A. Bonorino & Pchuajó & ", & 84.250 & 65.5 \\
\hline 81 & , & " & Salabcrry, Lalor y Bercetche & Laboulaye & Córrloba & 84.150 & 65.4 \\
\hline 82 & , & , & Chillado Genta y Cia. & Bolivar & Bucnos Aircs & $87 .-$ & 67.6 \\
\hline 83 & $\therefore$ & ,. & Salaberry, Lalor y Bctcetche & Gencral Soler & Córdoba & 84. - & 65.3 \\
\hline 84 & , & , & Pedro Estanguet & Laboulaye & , & 84.950 & $66 .-$ \\
\hline
\end{tabular}




\begin{tabular}{|c|c|c|c|c|c|c|c|}
\hline \multirow{2}{*}{ No. } & \multirow{2}{*}{\multicolumn{2}{|c|}{ PRODUCT }} & \multirow{2}{*}{$E X H|B| T O R$} & \multicolumn{2}{|c|}{ PLACE OF PRODUCTION } & \multirow{2}{*}{$\begin{array}{c}\text { Kilos } \\
\text { per } \\
\text { Hectolitre }\end{array}$} & \multirow{2}{*}{\begin{tabular}{|c} 
Lbs. \\
per \\
Bushel \\
(1)'CRESTER)
\end{tabular}} \\
\hline & & & & DISTRICT & PROVINCE & & \\
\hline 85 & Barletta & Wheat & José M. Mendez & Bonifacio & Buchos Aires & 83.450 & 64.5 \\
\hline 86 & , & $"$ & Pcdro Etchart & Toro &, & 83.350 & 64.7 \\
\hline 87 & , & ," & Balleto y Biclart & Chiclana &, & 84.950 & $66 .-$ \\
\hline 88 & , & $"$ & Devoto Balbiani y Cia. & Chivilcoy & , & $84.1 \overline{0} 0$ & 65.4 \\
\hline 89 & , & " & L. Lascaray & Mackenna & , & 82.400 & $64-$ \\
\hline 90 & , & $"$ & Juan Cañás & Colonia Cañas & Santa Fé & 84.950 & $66 .-$ \\
\hline 91 & " & $"$ & Clillado, Genta y Cia. & Bolivar & Buenos Aires & 84.950 & $66 .-$ \\
\hline 92 & , & " & Ginocchio, Etcheverry y Cia & Lanadrid & , & 85.150 & 66.2 \\
\hline 93 & " & $"$ & Ëscudero Hnos. & Lit Moro & , & 86.100 & 66.9 \\
\hline 94 & ," & $"$ & Löwengard y Cia. & L.os Toldos & ", & 81.700 & 63.5 \\
\hline 95 & , & $"$ & Devoto, Balbiani y Cia. & Dussaud & , & 84.700 & 65.8 \\
\hline 96 & ," & $"$ & Escudero Hnos. & Monte & , & s5. - & $66 .-$ \\
\hline 97 & $"$ & ; & Escudero Huos. & Rosclli & , & 85.150 & 66.2 \\
\hline 98 & ," & $"$ & Drabble Hnos. & Gcneral Villegas & , & $84 .-$ & 65.3 \\
\hline 99 & ", & $"$ & Ginocchio, Etcheverry y Cia. & Puan & , & 82.200 & 63.8 \\
\hline 100 & $"$ & ," & Balleto y Biclart & Chiclana & $"$ & 83.700 & $65 .-$ \\
\hline 101 & , & , & A. Velaz y Cia. & La Flora & , & 83. - & 64.5 \\
\hline 102 & , & , & Genoud, Benrenuto, Martelli y Cia. & Necocliea & , & 83.700 & $65 .-$ \\
\hline 103 & , & ", & Balleto y Bidart & San Perro & , & $\$ 3.350$ & 64.7 \\
\hline $10+$ & ," & ," & Fiorito y Cia. & $"$ & $"$ & $\$ 2.850$ & 64.4 \\
\hline 105 & , &, & Francisco Cianti & 9 de Julio & , & $8+.250$ & 65.5 \\
\hline 106 & " &, & Lucione y Ferrari & Giles &, & 83.450 & 64.8 \\
\hline 107 & $"$ & , & Palacios Hnos. & 9 de Julio &, & 84.500 & 65.6 \\
\hline 108 & $"$ & $"$ & Juan Barra & Castcllanos & Santa Fé & 83.550 & 64.9 \\
\hline 109 & , & $"$ & Natalio Vaberna & Las Colonias & $"$ & 83.200 & 64.6 \\
\hline 110 & , & ," & Eduardo Gochevind & San Carlos Sud & , & 82.750 & 64.3 \\
\hline 111 & $"$ & , & I. B. Baumann & Unión & Cordoba & 84. - & 65.3 \\
\hline 112 & , & $"$ & Marta de Hugucnch & Las Colonias & Santa $\mathrm{F}^{\prime} \mathrm{c}$ & 83.100 & 64.6 \\
\hline 113 & ," & ," & Ernesto Novillo & Alejo Ledesma & Cordoba & $84 .-$ & 65.3 \\
\hline 114 & ", & , & Bru110 y Cia. & Unión & ", & 84.950 & $66 .-$ \\
\hline 115 & ," & ,. & Felipe Carignano & San Justo &, & $84 .-$ & 65.3 \\
\hline 116 & , & ", & Viuda de C. Alcssio & Castellanos & Santa Fé & 84.350 & 65.5 \\
\hline 117 & , &, & Benito Villanueva & Lincoln & Buenos Aires & 83.200 & 64.6 \\
\hline 118 & ", & ," & D. Pirovano Hnos. & Castellanos & Santa Fé & 83.550 & 64.9 \\
\hline 119 & , & $"$ & Esteba11 Bonino & ", & ," & 83.450 & 64.9 \\
\hline 120 & , &, & Marco Zanni & General Lopez & ," & 83.550 & 64.9 \\
\hline 121 & , & , & Ayne Andrea & San Justo & Cordoba & 82.650 & 64.2 \\
\hline 122 & " & $\cdot$ & Antonio Lovato & La Capital & Santa Fé & 83.700 & $65 .-$ \\
\hline 123 & $"$ & ," & Juan Fraser & 9 de Julio & Buenos Aires & $8+.900$ & 65.9 \\
\hline 124 & ," & ", & Bernardo Angelini & Paraná & Entrc Rios & 82.050 & 63.7 \\
\hline 125 & " & $"$ & Vicente Colli & Bahía Blanca & Buenos Aires & 83.100 & 64.6 \\
\hline 126 & $"$ & ", & Carlomagno Hnos. & Unión & Cordoba & $\$ 5.150$ & 66.2 \\
\hline
\end{tabular}




\begin{tabular}{|c|c|c|c|c|c|c|c|}
\hline \multirow{2}{*}{ No. } & \multirow{2}{*}{\multicolumn{2}{|c|}{ PRODUCT }} & \multirow{2}{*}{$E X H I B \mid T O R$} & \multicolumn{2}{|c|}{ PLACE OF PRODUCTION } & \multirow{2}{*}{$\begin{array}{c}\text { Kilos } \\
\text { per } \\
\text { Hectolitre }\end{array}$} & \multirow{2}{*}{$\begin{array}{c}\text { Lbs. } \\
\text { per } \\
\text { Bushel } \\
\text { (II'CHESTER) }\end{array}$} \\
\hline & & & & DISTRICT & PROVINCE & & \\
\hline 127 & Barletta W & Wheat & Juan A. Brown & General Pinto & Buenos Aires & 85.4 .00 & 66.3 \\
\hline 128 & , &, & José Roscelli & Merlo & , & 83.200 & 64.6 \\
\hline 129 & ," & , & Gustavo Helmbold & Las Colonias & Santa Fé & 83.550 & 64.9 \\
\hline 130 & , & , & Máximo Paz & Constitución & , & 84.700 & 65.8 \\
\hline 131 & , & , & Raimundo Salazar y Cia. & F. Madero & Buenos Aires & 85.650 & 66.5 \\
\hline 132 & , &, & Carlos Valerotti & San Cristobal & Santa Fé & 84.150 & 65.4 \\
\hline 133 & $"$ &, & Groezinger Hnos. & San Martin & ", & 84.700 & 65.8 \\
\hline 134 & . & ," & Mateo Picoto & Las Colonias & $"$ & 83.550 & 64.9 \\
\hline 135 & , & , & Pablo Barbero & General Pinto & Buenos Aires & 83.200 & 64.6 \\
\hline 136 & $"$ & ," & Siro Capitini & Lincoln & $"$ & 84.850 & 65.9 \\
\hline 137 & , & , & Domingo Bauduco & Las Colonias & Santa Fé & 82.750 & 64.3 \\
\hline 138 & , & , & Cayetano De Vecehi & Tres Arroyos & Buenos Aires & 83.900 & 65.2 \\
\hline 139 & $"$ & , & Cirilo Marqui & Rosario Tala & Entre Ríos & 83.550 & 64.9 \\
\hline 140 & " & , & Gaston Hnos. & General Lopez & Santa Fé & 85.400 & 66.3 \\
\hline 141 & $"$ & , & Juan Gareía & San Martin &, & 83.800 & 65.1 \\
\hline 142 & ,. & , & Angel Curti & Castellanos &, & 82.550 & 64.1 \\
\hline 143 & $"$ & $"$ & José Bianconi & Constitución &, & 82.750 & 64.3 \\
\hline 144 & $"$ & , & Santiago Ferrero & J. Celman & Cordoba & 84.950 & $66 .-$ \\
\hline 145 & " & , & Natalio Mileri & Las Colonias & Santa Fé & 81.950 & 63.6 \\
\hline 146 & $"$ & , & Sebastian Maudeil & San Cristobal &, & 82.850 & 64.4 \\
\hline 147 & ," & , & S. Unzné é hijos & Pirovano & Buenos Aires & 83.700 & 65.- \\
\hline 148 &, & , & Figari y Camarero & Lincoln & , & 83.700 & 65.- \\
\hline 149 & , & , & Bunge y Born & Las Colonias & Santa Fé & 84.250 & 65.5 \\
\hline 150 & , & ", & E. Bonifacio & Bonifacio & Buenos Aires & 83.800 & 65.1 \\
\hline 151 & , & ", & Cirilo Odi & General Villegas & $"$ & $86 .-$ & 66.8 \\
\hline 152 & , & $"$ & Perpétuo Núñez & Azul &, & 82.750 & 64.3 \\
\hline 153 & , & $"$ & Carlos Rosetti & Genera1 Lopez & Santa Fé & 84.350 & 65.5 \\
\hline 154 & $"$ & $"$ & Estanguet y Salaberry & J. Celman & Cordoba & $82.050 \mid$ & $63-7$ \\
\hline 155 & , & , & Alberio E. Reffino & Paraná & Entre Ríos & 82.300 & 63.9 \\
\hline 156 & $"$ & , & Pedro Godino & Castellanos & Santa Fé & 84.250 & 65.5 \\
\hline 157 & , & , & Luis Ballaco & San Nicolás & Buenos Aires & 82.400 & $64 .-$ \\
\hline 158 & , & , & Barbeti Hnos. & Caseros & Santa Fé & 84.150 & 65.4 \\
\hline 159 & $"$ & , & Ricardo Petigreco & Bahía Blanea & Buenos Aires & 85.300 & 66.3 \\
\hline 160 & $"$ & $"$ & B. H. Lanfranchi & San Justo & Cordoba & 84.250 & 65.5 \\
\hline 161 & , & $"$ & Angel Motto & Villa Constitución & Santa Fé & 80.900 & 62.9 \\
\hline 162 & ", & $"$ & Ginoldi y Mano & Constitución & , & 82.200 & 63.8 \\
\hline 163 & $"$ & $"$ & Antonio Alisio & San Cristobal & , & 82.750 & 64.3 \\
\hline 164 & , & $"$ & Bautista Tauler & Río Cuarto & Cordoba & 85.150 & 66.2 \\
\hline 165 & , & " & Otto Widder & Las Colonias & Santa Fé & 84.350 & 65.5 \\
\hline 166 & , & , & Pio Ferrurese & J. Celman & Cordoba & 83.100 & 64.6 \\
\hline 167 & ", & " & Pedro Estanguet & , & $"$ & 85.850 & 66.7 \\
\hline 168 & $"$ & , & Juan B. Pinaqui & Laprida & Buenos Aires & 81.400 & 63.2 \\
\hline
\end{tabular}




\begin{tabular}{|c|c|c|c|c|c|c|c|}
\hline \multirow{2}{*}{ No. } & \multirow{2}{*}{\multicolumn{2}{|c|}{ PRODUCT }} & \multirow{2}{*}{ EXHIBITOR } & \multicolumn{2}{|c|}{ PLACE OF PRODUCTION } & \multirow{2}{*}{$\begin{array}{c}\text { Kilos } \\
\text { per } \\
\text { Hectolitre }\end{array}$} & \multirow{2}{*}{$\begin{array}{c}\text { Lbs. } \\
\text { per } \\
\text { Bushel } \\
\text { (IN'CllESTER) }\end{array}$} \\
\hline & & & & DISTRICT & PROVINCE & & \\
\hline 169 & Barletta & Wheat & Domingo Verte & Río Cuarto & Cordoba & 84.950 & 66.- \\
\hline 170 & ", & ", & Francisco Rivero & General Villegas & Buenos Aires & 85.050 & 66.1 \\
\hline 171 & $"$ & ", & José Sevese & San Martin & Santa Fé & 84.850 & 65.9 \\
\hline 172 & ," & $"$ & Migucl Ferrero & Castcllanos & $"$ & 82.650 & 64.2 \\
\hline 173 & ", & $"$ & Juan Beaupuy & $"$ & $"$ & 83.350 & 64.7 \\
\hline 174 & $"$ & " & Martin Bhuyre & Saavedra & Buenos Aires & 85.750 & 66.6 \\
\hline 175 & ," & $"$ & Juan Bianchi & General Pinto & " & 87.150 & 67.7 \\
\hline 176 & $"$ & ", & Pablo Ventersini & Saavedra & ", & 86.650 & 67.3 \\
\hline 177 & ", & $"$ & Juan Beaupuy & Castellanos & Santa Fé & 83.700 & 65.- \\
\hline 178 & ", & , & Juan Hampson & Bahía Blanca & Buenos Aires & 83.550 & 64.9 \\
\hline 179 & $"$ & $"$ & José Gieco & San Gcrónimı & Santa Fé & 83.100 & 64.6 \\
\hline 180 & $"$ & $"$ & Pedro Caballero & Las Colonias & $"$ & 82.650 & 64.2 \\
\hline 181 & , & $"$ & Ignacio Guraya & San Lorenzo & $"$ & 84.150 & 65.4 \\
\hline 182 & $"$ & ", & Angel Bridorolle & Río Cuarto & Cordoba & 86.200 & 67.- \\
\hline 183 & $"$ & $"$ & Luis Mauro & Ca. Irigoyen & Santa Fé & 85.050 & 66.1 \\
\hline 184 & $"$ & $"$ & Vicente Barrale & Castellanos & $"$ & 84.500 & 65.6 \\
\hline 185 & $"$ & $"$ & Juan B. Arana & Pergamino & Bucnos Aircs & 82.750 & 64.3 \\
\hline 186 & ", & $"$ & Cristóbal Zatti & Paraná & Entre Ríos & 82.400 & $64 .-$ \\
\hline 187 & $"$ & $"$ & Julio Gravius & General Villegas & Bucnos Aircs & 85.850 & 66.7 \\
\hline 188 & $"$ & ", & Julian Miganne & Coronel Pringles & $"$ & 83.900 & 65.2 \\
\hline 189 & $"$ & $"$ & José Rososchi & Trenque Lauquen & ", & 81.950 & 63.6 \\
\hline 190 & $"$ & $"$ & Francisco Mairini & Río Cuarto & Cordoba & $83 .-1$ & 64.5 \\
\hline 191 & $"$ & ", & Victorio D. Honenzi & San Martin & Santa Fé & 84.500 & 65.6 \\
\hline 192 & $"$ & $"$ & Antonio Lingua & San Cristobal & $"$ & 85.050 & 66.1 \\
\hline 193 & $"$ & ", & $J \cdot$ y V. Antuña & Las Colonias & $"$ & 84.500 & 65.6 \\
\hline 194 & ", & , & Carlos C. Olivera ć hijos & Necochea & Buenos Aires & 83.800 & 65.1 \\
\hline 195 & $"$ & $"$ & Bautista Moriena & Iriondo & Santa Fć & 83.900 & 65.2 \\
\hline 196 & ," & ," & Salomón Maldonado & General Lopez & $"$ & 82.750 & 64.3 \\
\hline 197 & $"$ & $"$ & Luis Serrano & Bahía Blanca & Bucnos Aires & 84.600 & 65.7 \\
\hline 198 & " & ", & Martin Badalloti & San Lorenzo & Santa Fé & 85.050 & 66.1 \\
\hline 199 & ," & ," & Lorenzo Giacosa & Sa11 Gerónimo & " & 85.650 & 66.5 \\
\hline 200 & ", & ", & Pablo Venturini & Saavedra & Bucnos Aires & 84.850 & 65.9 \\
\hline 201 & , & , & Bertossi y Oldani & Las Colonias & Santa Fé & 84.350 & 65.5 \\
\hline 202 & ", & $"$ & Cristóbal Prass & Paraná & Entre Ríos & 81.500 & 63.3 \\
\hline 203 & $"$ & , & Baratcabal y Ciraco & Lobería & Buenos Aires & 82.050 & 67.7 \\
\hline 204 & $"$ & $"$ & Ambrosio Baudino & San Martin & Santa Fé & 83.100 & 64.6 \\
\hline 205 & , & $"$ & Miguel Eizmendi & Río $3^{\circ}$ Arriba & Cordoba & 85.400 & 63.3 \\
\hline 206 & $"$ & $"$ & Antonio Calalde & Río $3^{\circ}$ A bajo & $"$ & 81.850 & 63.6 \\
\hline 207 & ", & $"$ & Alvarez Hnos. & Chacabuco & Buenos Aires & 83.350 & 64.7 \\
\hline 208 & $"$ & $"$ & Maria D. de Martin & Conc. del Uruguay & Entre Ríos & 83.100 & 64.6 \\
\hline 209 & $"$ & $"$ & Salvador Bosque & Iriondo & Santa Fé & 82.700 & 64.3 \\
\hline 210 & $"$ & $"$ & Pascuale R. Olivo & Castellanos & " & 83.200 & $64: 6$ \\
\hline
\end{tabular}




\begin{tabular}{|c|c|c|c|c|c|c|c|}
\hline \multirow{2}{*}{ No. } & \multirow{2}{*}{\multicolumn{2}{|c|}{ PRODUCT }} & \multirow{2}{*}{$E X H I B I T O R$} & \multicolumn{2}{|c|}{ PLACE OF PRODUCTION } & \multirow{2}{*}{$\begin{array}{c}\text { Kilos } \\
\text { per } \\
\text { Hectolitre }\end{array}$} & \multirow{2}{*}{$\begin{array}{c}\text { Lbs. } \\
\text { per } \\
\text { Bushel } \\
\text { (W'CllESTER) }\end{array}$} \\
\hline & & & & DISTRICT & PROVINCE & & \\
\hline 211 & Barletta & Wheat & Antonio Serasio & Belgrano & Santa Fé & 84.250 & 65.5 \\
\hline 212 & ", & ," & Bautista Bonzo & Gencral Pinto & Bucnos Aires & 85.500 & 66.4 \\
\hline 213 & , & , & F. y G. Griffa & Castellanos & Santa Fé & 83.100 & 64.6 \\
\hline 214 & . & " & Alcjandro Minerdi & ", & $"$ & 83.350 & 64.7 \\
\hline 215 & , & , & Roque Feyrrera & Río $3^{\circ}$ Abajo & Cordoba & 84.150 & 65.4 \\
\hline 216 & , &, & Dario Garcia & Coronel Dorrego & Bucnos Aires & 83.200 & 64.6 \\
\hline 217 & ," & , & Fernando Favre & Las Colonias & Santa Fé & 82.650 & 64.2 \\
\hline 218 & ", & ", & Juaı Elcna & Colón & Cordoba & 81.250 & 63.1 \\
\hline 219 & ", & ", & Francisco Gotte & Paraná & Entre Ríos & 82.550 & 64,1 \\
\hline 220 & ", & ," & Depetre Hnos. & General Pinto & Bucnos Aires & 86.100 & 66.9 \\
\hline 221 & , & ," & A. Demarchi & San Martin & Santa Fé & 82.050 & 63.7 \\
\hline 222 & $"$ & ," & Alfredo Beltrame & Belgrano & ", & 83.800 & 65.1 \\
\hline 223 & $"$ & , & Pedro Aicardi & San Martin & , & 84.500 & 65.6 \\
\hline 224 & , & , & Alvarez Hnos. & Chacabuco & Buenos Aires & 83.350 & 64.7 \\
\hline 225 & , & , & Ormaechea Hnos. & 9 de Julio &, & 84.350 & 65.5 \\
\hline 226 & , & , & Braulio Nieto & Gilcs & , & 82.300 & 63.9 \\
\hline 227 & $"$ & , & Matía Hnos. & Río Segundo & Cordoba & 84. - & 65.3 \\
\hline 228 & , & , & Juan Brusa & , & , & 84. - & 65.3 \\
\hline 229 & $"$ & ," & Augusto Sixte & Puan & Buenos Aires & 84.250 & 65.5 \\
\hline 230 & $"$ & ," & Juan Pinasco & Cascros & Santa Fé & 84.850 & 65.9 \\
\hline 231 & , & ," & Sebastian Zocola & M. Juarez & Cordoba & 83.700 & $65 .-$ \\
\hline 232 & , &, & Pablo Hofstaetter & Diamante & Entre Ríos & 83.200 & 64.6 \\
\hline 233 & $"$ & ," & Rodolfo Peattine & Río $3^{\circ}$ Abajo & Cordoba & 82.050 & 63.7 \\
\hline 234 & $"$ &, & Agustin Eloriaga & A. Alsina & Bucnos Aires & 82.650 & 64.2 \\
\hline 235 & ., & , & Leopoldo Ghezzi & Castellanos & Santa Fé & 83.350 & 64.3 \\
\hline 236 & ," &, & Domingo E. Rodriguez & Tres Arroyos & Bucnos Aires & 83.200 & 64.6 \\
\hline 237 & Hungarian & Wheat & Palacios Hiros. & Carhué & , & 84.250 & 65.5 \\
\hline 238 & , & , & C. Grittini & Saladillo & , & 84.350 & 66.5 \\
\hline 239 & ", & , & S. Koppi y Cia. & Islas &, & 82.400 & $64 .-$ \\
\hline 240 & ", & , & Ojea y Garcia & Pàso & ," & 82.400 & $64 .-$ \\
\hline 24.1 &, & , & Santamarina é hijos & Pehuajó & , & 82.900 & 64.4 \\
\hline 242 & ", & , & Ojea y García & Passo & , & 84. - & 65.3 \\
\hline 243 & , & , & Salaberry, Lalor y Bercetche & Las Flores & , & 84.600 & 65.7 \\
\hline 244 & ," & ", & D. Boubier & Pehuajó & , & 83.350 & 64.7 \\
\hline 245 & , & , & Devoto, Balbiani y Cia. & La Zanja & " & 82.850 & 64.4 \\
\hline 246 & , & ," & L. Dreyfus & Junin & , & 83.550 & 64.9 \\
\hline 247 & ," & $"$ & Ojea \& García & Mercedes & ,. & 83.550 & 64.9 \\
\hline 248 & , & $"$ & Juan Devoto (hijo) & 9 de Julio & ," & 82.650 & 64.2 \\
\hline 249 & , & " & B. Bonorino & General Villegas & , & 84.600 & 65.7 \\
\hline 250 & , & , & Devoto, Balbiani y Cia. & Drysdale &, & 85.150 & 66.2 \\
\hline 251 & $"$ & ," & S. Unzué é hijos & M. Michel & ", & 82.650 & 64.2 \\
\hline 252 & ," & , & A. Drabble & Gencral Villegas & ", & $\$ 3.550$ & 64.9 \\
\hline
\end{tabular}




\begin{tabular}{|c|c|c|c|c|c|c|c|}
\hline \multirow{2}{*}{ No. } & \multirow{2}{*}{\multicolumn{2}{|c|}{ PRODUCT }} & \multirow{2}{*}{ EXHIBITOR } & \multicolumn{2}{|c|}{ PLACE OF PRODUCTION } & \multirow{2}{*}{$\begin{array}{c}\text { Kilos } \\
\text { per } \\
\text { Hectolitre }\end{array}$} & \multirow{2}{*}{$\begin{array}{c}\text { Lbs. } \\
\text { per } \\
\text { Bushel } \\
\text { (W'Cllester) }\end{array}$} \\
\hline & & & & DISTRICT & PROVINCE & & \\
\hline 253 & Hungarian & Wheat & Nogucras y Avalos & F. Madero & Buenos Aires & 85.400 & 66.3 \\
\hline $25+$ & ," & ," & Ojea y García & Passo & ," & 82.300 & 63.9 \\
\hline 255 & ," & " & Palacios Hnos. & ," & ", & 81.500 & 63.3 \\
\hline 256 & ," & ," & Deroto, Balbiani y Cia. & Suipacha & ," & 83.100 & 64.6 \\
\hline 257 & ", & ", & Palacios Inos. & 9 de Julio & ," & 83.450 & 64.8 \\
\hline 258 & ," & , & Devoto, Balbiani y Cia. & F. Madero & ," & 82.650 & $6+.2$ \\
\hline 259 & ," & ," & Unzué é hijos & Cambaceres & ," & 84.350 & 6.5 .5 \\
\hline 260 & , & ," & Orcoyen, Castaños y Cia. & Pehuajó & ," & 83.100 & 64.6 \\
\hline 261 & , & ," & S. Zoppi y Cia. & $"$ & ," & 82.400 & $64 .-$ \\
\hline 262 & ", &, & Ojea y García & Passo & , & 82.300 & 63.9 \\
\hline 263 &, & ," & , & Drysdale & ," & 82.050 & 63.7 \\
\hline 264 & , & $"$ & Santamarina é hijos & Chiclana & " & 83. - & 64.5 \\
\hline 265 & ," & ," & Juan Devoto (hijo) & 9 de Julio & ," & 81.150 & 63.- \\
\hline 266 & ," & ", & Orcoyen, Castaños y Cia. & F. Madero & ," & 83. - & 64.5 \\
\hline 267 &, &, & E. Greslevin & Gencral Uriburu &. & 82.050 & 63.7 \\
\hline 268 & ," &, & Ojea y García & $J$ unin & , & 83.100 & 64.6 \\
\hline 269 & ," & ", & Santamarina é hijos & Pehuajó & , & 84.950 & $66 .-$ \\
\hline 270 & ," & ," & Ojea y García & Junin & " & 84.950 & 66.- \\
\hline 271 & , & ," & Ginocchio, Etcheverry y Cia. & Pehuajó & $"$ & 81.700 & 63.5 \\
\hline $2 \pi 2$ & , & , & H. Heiggines & Cambaceres & $"$ & 82.050 & 63.7 \\
\hline 273 &, &, & Nogueras y Avalos & F. Madero & ", & 82.750 & 64.3 \\
\hline 274 & ", & ," & Lucione y Ferrari & Cucullú & ", & 83.350 & 64.7 \\
\hline 275 &, & ", & L. Dreyfus & Passo & ," & 82.300 & 63.9 \\
\hline 276 & ," &, & Dcroto, Balbiani y Cia. & Gencral Chaves & ", & 83. -1 & 64.5 \\
\hline 277 & ," & ", & Ginocchio, Etcheverry y Cia. & Pehuajó & , & 81.600 & 63.4 \\
\hline 278 & ," & ," & Palacios Hnos. & 9 de Julio & ", & 83.450 & 64.8 \\
\hline 279 & ", &, & S. Unzué é hijos & M. Fernandez & ", & 84.250 & 65.5 \\
\hline 280 & ", & ,. & L. Dreyfus & Gencral Las Heras & ", & 82.4 .00 & $64 .-$ \\
\hline 281 & " & ," & P. V. Jauregui & Trenque Lauquen & ," & 84.350 & 65.5 \\
\hline 282 &. & $"$ & Domingo Galli & Las Colonias & Santa Fé & 83.800 . & 65.1 \\
\hline 283 & ," & " & Antonio Cocco & Río Cuarto & Cordoba & 86.300 & $67 .-$ \\
\hline 284 & , & ," & Pedro Etchart & Trenque Lanquen & Bucnos Aires & 84.850 & 65.9 \\
\hline 285 & ", & ", & Ojea y García & Drysdale & ", & 83.900 & 65.2 \\
\hline 286 & ", & , & Devoto, Balbiani y Cia. & Disssaud & , & 84. - - & 65.3 \\
\hline 287 & ," & ," & Isidoro Chervaz & Baradero & ," & 83. - - & 64.5 \\
\hline 288 & ," & ," & Ojea y García & Dussaud & ," & 83.350 & 64.7 \\
\hline 289 & ," & , & Juan Maupas & 9 de Julio & , & 84.850 & 65.9 \\
\hline 290 & ," & ," & Genoud, Benvenuto, Martelli y Cia. & Arenales & ", & 83.4 .50 & 64.8 \\
\hline 291 & , & ," & Patricio Ham & Chiclana & ,. & 82.400 & 64.- \\
\hline 292 & ," & , & S. Unzué é hijos & Lincoln & ", & $84.2 \tilde{50}$ & 65.5 \\
\hline 293 & ", & $"$ & J. Navarro & , & $"$ & 84.600 & 65.7 \\
\hline 294 & , & $"$ & Lucione $y$ Ferrari & Banderaló & $"$ & 83.350 & 64.7 \\
\hline
\end{tabular}




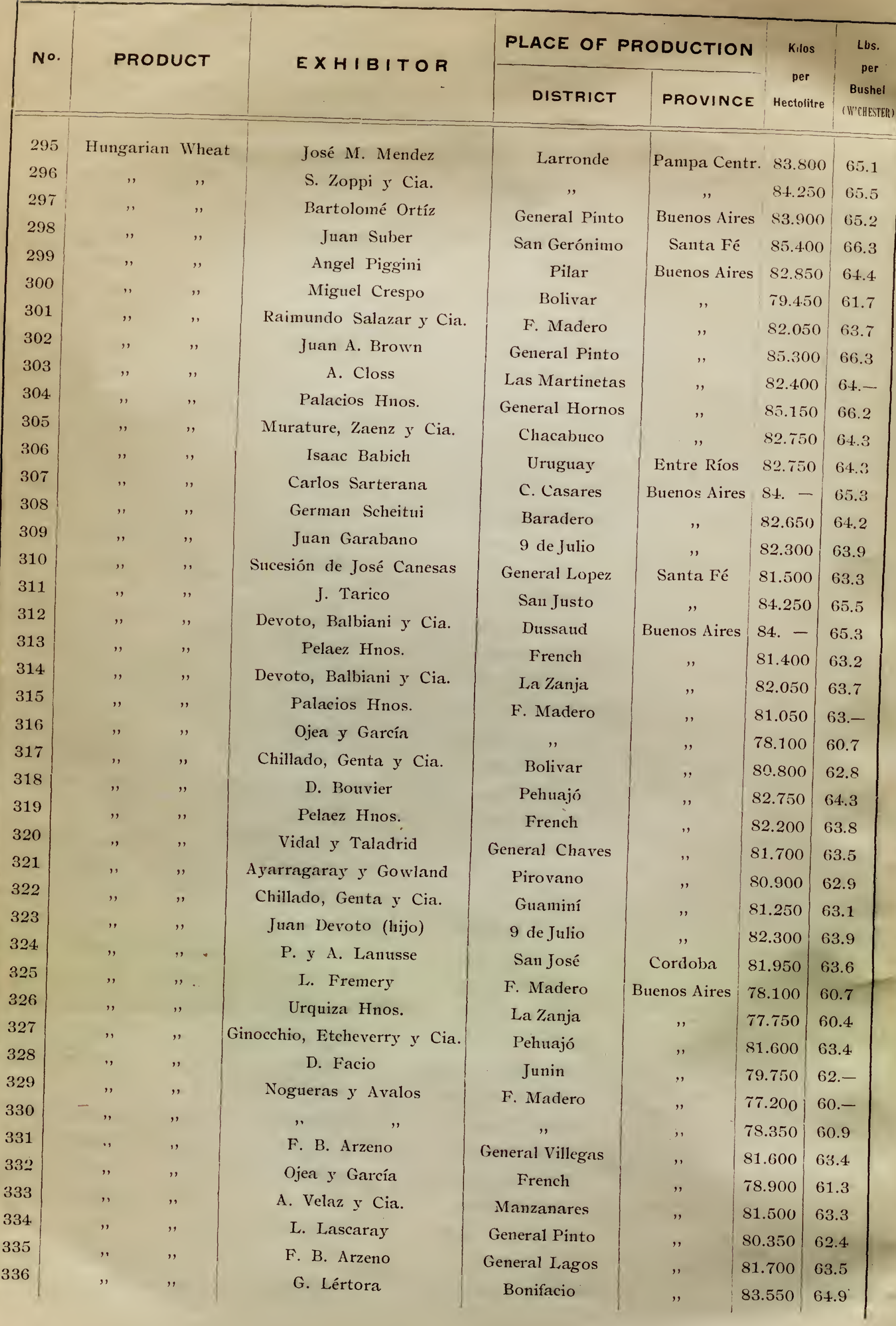




\begin{tabular}{|c|c|c|c|c|c|c|c|}
\hline \multirow{2}{*}{ No. } & \multirow{2}{*}{\multicolumn{2}{|c|}{ PRODUCT }} & \multirow{2}{*}{ EXHIBITOR } & \multicolumn{2}{|c|}{ PLACE OF PRODUCTION } & \multirow{2}{*}{$\begin{array}{c}\text { Kilos } \\
\text { per } \\
\text { Hectolitre }\end{array}$} & \multirow{2}{*}{$\begin{array}{c}\text { Lbs. } \\
\text { per } \\
\text { Bushel } \\
\text { (W'CHESTER) }\end{array}$} \\
\hline & & & & DISTRICT & PROVINCE & & \\
\hline 673 & Russian W & Wheat & Bassi Hnos. & La Flora & Buenos Aires & 83.550 & 64.9 \\
\hline 674 & $"$ & ". & Juan Crupre & 25 de Mayo & ", & 81.400 & 63.2 \\
\hline 675 & ," &.$\dot{.}$ & "Curumalan", Company & Coronel Suarez & $"$ & 85.050 & 66.1 \\
\hline 676 & ", & " & Pcdro Madler & General Lamadrid & " & 83.700 & $65 .-$ \\
\hline 677 & $"$ & . & S. Estibe y Cía. & Pehıajó & ", & 84.250 & 65.5 \\
\hline 678 & Anchuclo W & Wheat & Giovanini Fonatti & San Cristóbal & Santa Fé & 82.550 & 64.1 \\
\hline 679 & ," & " & Juan Galizzi Hno. & Nogoyá & Entre Ríos & 82.400 & $64 .-$ \\
\hline 680 & , & ,. & Luis Gondini & Castellanos & Santa Fé & 84.500 & 65.6 \\
\hline 681 & , & $"$ & José Restelli & Las Colonias & $"$ & 86.300 & $67 .-$ \\
\hline 682 & ", & $"$ & Luis Schmidt & Paraná & Entre Ríos & 81.950 & 63.6 \\
\hline 683 & , & ,. & Jorge Boaglis & Nogoyá & , & 81.400 & 63.2 \\
\hline 684 & , ' & , & Albino E. Reffino & Paraná & , & 82.750 & 64.3 \\
\hline 685 & ". & .. & J. I. Waigaudt & Diamante & ," & 84.150 & 65.4 \\
\hline 686 & $"$ & $"$ & Carlos Pcnatti, & Nogoyá & , & 83.800 & 65.1 \\
\hline 687 & $"$ & $"$ & Mateo Dorao & Capital & S. del Estero & 80.700 & 62.7 \\
\hline 688 & Anchuelo Whit & te Wheat & $"$ & , & $"$ & 77.550 & 60.2 \\
\hline 689 & Anchuelo W & Wheat & Angel Corbetto & Colón & Entre Ríos & 83.850 & 64.4 \\
\hline 690 & , , & , & Francisco Gandini & Castellanos & Santa I'é & 83.850 & 64.4 \\
\hline 691 & Hungarian & Wheat & Salvador Busso & Lincoln & Buenos Aires & 85.050 & 66.1 \\
\hline 692 & ," & , & Inocencio Buffa Hnos. & San Isidro & $"$ & 81.500 & 63.3 \\
\hline 693 & $"$ & , & Antonio D'Ambrosio & Trenque Lauquen & $"$ & 80.200 & 62.3 \\
\hline 694 & ," & , & Bassi Hnos. & La Flora & , & 83.800 & 65.1 \\
\hline 695 & , & ", & Issa y Díaz & Pehuajó & ," & 83.100 & 64.6 \\
\hline 696 & Piedmontese & Wheat & Antonio D'Ambrosio & Trenquc Lauquen & " & 82.550 & 64.1 \\
\hline 697 & ", &, & Bassi Hnos. & Las Flores & $"$ & 83.550 & 64.9 \\
\hline 698 & ," & ," & Juan F. Diaz & Pehuajó & ", & 83.200 & 64.6 \\
\hline 699 & Tusclas W] & Theat & Lucione y Ferrari & O'Higgins & $"$ & 83.550 & 61.- \\
\hline 700 & ," & , & José M. de la Barrera & Chivilcoy & $"$ & 80.100 & 62.2 \\
\hline 701 & ," & " & Antonio Demarchi & Pilar & $"$ & 80.900 & 62.9 \\
\hline 702 & ", & , & José Repetto & Chivilcoy & $"$ & 81.950 & 63.3 \\
\hline 703 & Berton & , & Josć Ghione & San Martin & Santa Fé & 80.550 & 62.6 \\
\hline 704 & $"$ & $"$ & Cesare Gioco & Diamante & Entre Ríos & 83.100 & 64.6 \\
\hline 705 & ", & $"$ & Lorenzo Gaido & San Martin & Santa Fé & 81.050 & 63.- \\
\hline 706 & , & $"$ & Avalle Hnos. & $"$ & ", & 80.700 & 62.7 \\
\hline 707 & , & $"$ & Emilio D. Ortiz & $"$ & ," & 80.800 & 62.8 \\
\hline 708 & ," & " & G. Ibeinych & San Cristobal & $"$ & 79.750 & 62.- \\
\hline 709 & Linseed & & Francisco Pegaz & Pergamino & Buenos Aires & 70.4 .00 & 54.7 \\
\hline 710 & , & & Antonio Demarchi & Pilar & ,. & 70.200 & 54.5 \\
\hline 711 & ; & & Luis Rosset & Rojas & ", & 70.500 & 54.8 \\
\hline 712 & ," & & José Musso & Pergainino & $"$ & $70 .-$ & 54.3 \\
\hline 713 & ", & & U. Buzzoni & Belgrano & Santa Fé & 69.300 & 53.8 \\
\hline 714 & $"$ & & Lucas Seulli & S. Antonio de Areco & Buenos Aires & $66 .-$ & 51.3 \\
\hline
\end{tabular}




\begin{tabular}{|c|c|c|c|c|c|c|}
\hline \multirow{2}{*}{ No. } & \multirow{2}{*}{ PRODUCT } & \multirow{2}{*}{ EX H IB I TOR } & \multicolumn{2}{|c|}{ PLACE OF PRODUCTION } & \multirow{2}{*}{$\begin{array}{c}\text { Kilos } \\
\text { per } \\
\text { Hectolitre }\end{array}$} & \multirow{2}{*}{$\begin{array}{c}\text { Lbs. } \\
\text { per } \\
\text { Bushel } \\
\text { (W'Cllestren) }\end{array}$} \\
\hline & & & DISTRICT & PROVINCE & & \\
\hline 715 & Linseed & Bartolo Bonino & Las Colonias & Santa Fé & 68.700 & 53.4 \\
\hline 716 & , & Alejo Botchatael & Colon & Entre Ríos & 68.400 & 53.1 \\
\hline 717 & , & Chiesa y Modachini & Lineoln & Buenos Aires & 70.300 & 54.6 \\
\hline 718 & , & Felipe Cosetti & Belgrano & Santa Fé & 68.500 & 53.2 \\
\hline 719 & ", & Juan M. Seró & Uruguay & Entre Ríos & 70.500 & 54.8 \\
\hline 720 & , & Colonia San Normando & San Martin & Santa Fé & 69.200 & 53.7 \\
\hline 721 & , & Carlos Falcone & Caseros & , & 69.300 & 53.8 \\
\hline 722 & , & José Severo & San Martin & , & 70.- & 54.3 \\
\hline 723 & , & Carlos A. Bueyo & Paraná & Entre Ríos & 70.400 & 54.7 \\
\hline 724 & , & Alcjandro Bedoni & S. Antonio de Arceo & Buenos Aires & 69.800 & 54.3 \\
\hline 725 & ,. & Segundo Giecco & Diamantc & Entre Ríos & 68.800 & 53.5 \\
\hline 726 & " & Luis Cliabone & Rosario Tala & ," & 69.700 & 54.1 \\
\hline 727 & , & Cías. Brinkmann, Seeber y Bottini & San Justo & Córdoba & 71.300 & 55.4 \\
\hline 728 & , & José Scagliotti & Belgrano & Santa Fé & 69.700 & 54.1 \\
\hline 729 & ", & José Roscclli & Merlo & Buenos Aires & 70.700 & $55 .-$ \\
\hline 730 &, & Juan M. Magnin & Colon & Entre Ríos & 67.200 & 52.2 \\
\hline 731 & ", & Gaston Hnos. & General Lopez & Santa Fé & 70.700 & $55 .-$ \\
\hline 732 & ", & Juan Pcrez & San Andrés de Giles & Buenos Aires & 69.700 & 54.1 \\
\hline$\tau 33$ & , & Luis Paladini & Caseros & Santa Fé & 70.250 & 54.6 \\
\hline 734 & $"$ & Antonio Zussero & V. Constitución & ,. & 70. & 54.3 \\
\hline 735 & , & Salomon Maldonado & General Lopez & , & 68.700 & 53.4 \\
\hline 736 & ", & N. Botta Hnos. & San Justo & , & 68.500 & 53.2 \\
\hline 737 & $"$ & Giecco é hijos & Diamantc & Entre Ríos & 69.400 & 53.9 \\
\hline 738 & , & P. Villagran & Paraná & , & 67.500 & 52.4 \\
\hline 739 & , & Groczinger Hnos. & San Martin & Santa Fé & 69.200 & 53.7 \\
\hline 740 & , & Enrique N. Larden & General Lopez & , & 66.500 & 51.7 \\
\hline 741 & , & Pedro Luro é hijos & Villarino & Buenos Aires & 67.800 & 52.7 \\
\hline 742 & , & A. Robledo & Pilar & , & 71. - & 55.1 \\
\hline 743 & , & Ojea y García & Mercedes & , & 71.300 & 55.4 \\
\hline 744 & , & Feliberto Pégaz & Pergamino & , & 69.500 & $54 .-$ \\
\hline 745 & , & José Franzoni & Rojas &, & 68.4 .00 & 53.1 \\
\hline 746 & , & Devoto, Balbiani y Cia. & Suipacha & , & 68.800 & 53.5 \\
\hline 74.7 & " & Porten Hnos. & San Pedro & , & $69 .-$ & 53.6 \\
\hline 74.8 & , & Mariano Lopez & Bragado &, & 68.800 & 53.5 \\
\hline 749 & $"$ & Juan Analozzi Hno. & Nogoyá & Entre Ríos & 69.200 & 53.7 \\
\hline 750 & , & P. C. Pera & General Pinto & Buenos Aires & 68. - & 52.8 \\
\hline 751 & $"$ & Juan Pinasco & Caseros & Santa Fé & 66.600 & 51.7 \\
\hline 752 & $"$ & Tersoglio $y$ de Angelis & Marcos Juarez & Córdoba & 70. & 54.3 \\
\hline 753 & , & E. Orsetto & Rosario & Santa Fé & 70.100 & 54.4 \\
\hline 754 & $"$ & Jorge Barbieh & Baradcro & Bucuos Aires & 70.500 & 54.8 \\
\hline 755 & , & Ernesto Pasetti & Gualeguaychú & Entre Ríos & 69.200 & 53.7 \\
\hline 756 & , & Antonio Calaldi & Río $3^{\circ}$ Abajo & Córdoba & 66.600 & 51.7 \\
\hline
\end{tabular}




\begin{tabular}{|c|c|c|c|c|c|c|}
\hline \multirow{2}{*}{ No. } & \multirow{2}{*}{ PRODUCT } & \multirow{2}{*}{$E X H|B| T O R$} & \multicolumn{2}{|c|}{ PLACE OF PRODUCTION } & \multirow{2}{*}{$\begin{array}{c}\text { Kilos } \\
\text { per } \\
\text { Hectolitre }\end{array}$} & \multirow{2}{*}{$\begin{array}{c}\text { Lbs. } \\
\text { per } \\
\text { Bushel } \\
\text { (W'CHBSTER) }\end{array}$} \\
\hline & & & DISTRICT & PROVINCE & & \\
\hline 757 & Linsced & Sebastian Caglieri & Castellanos & Santa Fé & 66.800 & 51.9 \\
\hline 758 & , & B. B. Taylor & Rosario Tala & Entrc Rios & 69.300 & 53.8 \\
\hline 759 & ", & Julian Agmaga & Iriondo & Santa Fé & 69.300 & 53.8 \\
\hline 760 & $"$ & A. $y$ B. Boero & San Justo & Córdoba & 69.300 & 53.8 \\
\hline 761 & ," & Felix Peretta & San Gcrónimo & Santa Fé & 70.100 & 54.4 \\
\hline 762 & , & Maria D. de Ranno & , &, & 70.400 & 54.7 \\
\hline 763 & $"$ & Lino Saccuzzi & Reconquista & ", & 69.600 & 54.1 \\
\hline 764 & ", & Felix Raimondi & San Gerónimo & ", & 70.900 & 55.- \\
\hline 765 & $"$ & B. R. Pérez & Carmen de Areco & Buenos Aires & 70.300 & 54.6 \\
\hline 766 & $"$ & Gianello y Bettarcl & Gualeguay & Entre Ríos & 68.700 & 53.4 \\
\hline 767 & ", & Murature, Saenz y Cía. & Suipacha & Buenos Aires & 71.800 & \begin{tabular}{|l|}
55.7 \\
\end{tabular} \\
\hline 768 & Oats & Baratebal y Ciraco & Loberia & ," & 51.650 & 40.1 \\
\hline 769 & ", & Alvarcz Hnos. & Chacabuco & ", & 49.850 & 38.7 \\
\hline 770 & , & Juan Albarello & Lincoln & ", & 39.800 & 30.8 \\
\hline 771 & , & Angel Mercoela & San Isidro & ," & 44.850 & 34.8 \\
\hline 772 & , & Paulino Rey & Bolivar & $"$ & 50.900 & 39.5 \\
\hline 773 & ", & Juan Parazzalo & Junin & , & 50.600 & 39.3 \\
\hline 774 & , & Ricardo Lavalle & Lobería &, & 41. - & 31.9 \\
\hline 775 & , & " $\quad$, & $"$ & ," & 43.100 & 33.5 \\
\hline 776 & ", & lauregui P. V. & Bayauca & ", & 46.700 & 36.3 \\
\hline 777 & $"$ & Roberto Cano & Merlo & $"$ & 43.300 & 33.6 \\
\hline 778 & $"$ & Sala de Comercio I1 de Sepbrc. & Villarino & $"$ & 44.850 & 34.8 \\
\hline 779 & , &,$\quad$, & ", & $"$ & 51.750 & 4.0 .2 \\
\hline 780 & , & Pedro Luro é Hijos & , & $"$ & 50.900 & 39.5 \\
\hline 781 & Barley & Chillado, Genta y Cia. & Carhuć & , & 62.200 & 48.3 \\
\hline 782 & ," & Corleto y Cia. & San Pedro & ," & 58.950 & 4.5 .8 \\
\hline 783 & Malting Barley & J. Goñi & Berutti & $"$ & 67.800 & 52.7 \\
\hline 784 & Barley & Vicente Colli & Bahía Blanca & $"$ & 67.800 & 52.7 \\
\hline 785 & , & P. y A. Lanusse & Las 3 Marías & , & 62.600 & 48.6 \\
\hline 786 & , & Dical Julio & Chacabuco & $"$ & 60.950 & 47.4 \\
\hline 787 & , & Cristobal Walcwcin & Puan & " & 57.750 & 44.9 \\
\hline 788 & $"$ & V. Colli & Bahia Blanca & , & 67.800 & 52.7 \\
\hline 789 & , & Ricardo Pettigrew & , & $"$ & - & - \\
\hline 790 & Rye grass & J. J. Alberdi & Coronel Suarez & $"$ & - & - \\
\hline 791 & $", \quad$, & Ricardo Lavalle & Lobería & ", & - & - \\
\hline 792 & Canary-seed & Francisco Pegaz & Pergamino & , & 74.600 & $58 .-$ \\
\hline 793 & ," & Domingo Carnevalle & La Capital & Santa Fé & 74. & 57.5 \\
\hline 794 & , & Francisco Armando & San Justo & ," & 72.200 & \begin{tabular}{|l|l|}
56.1 &
\end{tabular} \\
\hline 795 & ," & Gustavo Helmbold & Las Colonias & ," & 74.700 & $58 .-$ \\
\hline 796 & , & Pedro Bodanoch & Rosario & , & 74.700 & $58 .-$ \\
\hline 797 & , & Juan Traverso & $\mathrm{Paz}$ & Córdoba & 73.400 & $57 .-$ \\
\hline 798 & , & Roberto Cima & Colon! & Buenos Aires & 73.900 & 57.4 \\
\hline
\end{tabular}




\begin{tabular}{|c|c|c|c|c|c|c|c|}
\hline \multirow{2}{*}{ No. } & \multirow{2}{*}{ PRODUCT } & \multirow{2}{*}{\multicolumn{2}{|c|}{ EXHIBITOR }} & \multicolumn{2}{|c|}{ PLACE OF PRODUCTION } & \multirow{2}{*}{$\begin{array}{c}\text { Kilos } \\
\text { per } \\
\text { Hectotitre }\end{array}$} & \multirow{2}{*}{$\begin{array}{c}\text { Lbs. } \\
\text { per } \\
\text { Bushel } \\
\text { (I'CHESTER) }\end{array}$} \\
\hline & & & & DISTRICT & PROVINCE & & \\
\hline 799 & Canary-sced & José Ducerre & & Luján & Bucnos Aires & 73.500 & $5 \pi .1$ \\
\hline 800 & ," & Antonio Zusse & & Villa Constitución & Santa Fé & 76.300 & 59.3 \\
\hline 801 & ," & Leonardo Pcre & & 'Tres Arroyos & Buenos Aires & 72.800 & 56.6 \\
\hline 802 & ," & Juan Dosce & & Baradero & " & 75. & 58.3 \\
\hline 803 & ", & Sucesión de .I. Ca & & General Lopez & Santa Fé & 75.600 & 58.7 \\
\hline 804 &, & J. Mazzoni & & San Justo & ", & 73.850 & 57.3 \\
\hline 805 & ", & Juan Faehill & & Rojas & Buenos Aires & 71.400 & 55.5 \\
\hline 806 &, & Juan Oberti & & Junin & ,. & 73.500 & 57.1 \\
\hline 807 & ", & Isidro Suáre & & Carmen de Areco & ", & 75.800 & 58.9 \\
\hline 808 & Lacern & Sala de Comercio 11 & p'bre. & - & Pampa Centr. & (1) & - \\
\hline 809 & , & " " & “ & - & Cliubut & (2) & - \\
\hline 810 & , & " $" ،$ & “ & Gencral Lagos & Pampa Centr. & (3) & - \\
\hline 811 & ", & Raimundo Salazar & Cía. & F. Madero & Buenos Aires & (4) & - \\
\hline 812 & , & Capdevilla Alfo & & General Lagos & Panpa Contr & (5) & - \\
\hline 813 & , & “ & & , & ", & (6) & - \\
\hline 814 & , & B. G. Baluido & & T. Lauquen & Buenos Aires & ( $(\tau)$ & - \\
\hline 815 & Beans & Bernardo Saupt & & Las Flores & ," & 75.100 & 58.3 \\
\hline 816 &, & P. Dumont & & Cliascomús & ", & 76.300 & 59.3 \\
\hline 817 & " & Pio Ferrares & & J. Alman & Córdoba & 68.300 & 53.- \\
\hline 818 & $"$ & Domingo Sab: & & San Martin & Mendoza & 75.200 & 58.4 \\
\hline 819 & ", & Sala de Comereio 11 & ct'bre. & " & $"$ & 74.700 & 58.- \\
\hline 820 & ", & $"$ & " & ," & Buenos Aires & 72.700 & 56.4 \\
\hline 821 & , & $"$ & , & Mercedes & ", & 74.200 & 57.6 \\
\hline 822 & " & $"$ & , & " & $"$ & $73 .-$ & 56.7 \\
\hline 823 & , & ," & ", & ", & Santa Fé & 72.400 & 56.2 \\
\hline 824 & , &,$\quad$, & , & Baradero & Buenos Aires & 72. & 55.9 \\
\hline 825 & , & Vicente Alon & & Las Garzas & Santa Fé & $66 .-1$ & 51.3 \\
\hline 826 & $"$ & Sala de Comercio 11 & et'bre. & $"$ & , & 76.600 & 59.5 \\
\hline 827 & ", & ", & , & Chivilcoy & Buenos Aires & 75.300 & 58.5 \\
\hline 828 & Canary Beans & ," & $"$ & Saladillo & ," & 78.200 & 60.8 \\
\hline 829 & Flageolet „, & $"$ & $"$ & ", & $"$ & $76 .-$ & 59.- \\
\hline 830 & Beans & $"$ & $"$ & , & Santa Fé & 73. - & 56.7 \\
\hline 831 &, & , & ", & ," & Mendoza & 75.500 & 58.7 \\
\hline 832 & , & ", & " & , & Santa Fé & 74.500 & 57.8 \\
\hline 833 & $"$ & " & , & Lujan & Buenos Aires & 72.300 & 56.2 \\
\hline 834 & $"$ & $"$ & $"$ & ", & ", & 74.700 & 58.- \\
\hline 835 & $"$ & $"$ & , & ", & Santa Fé & 76.200 & 59.2 \\
\hline 836 & Broad Beans & ", & $"$ & ", & Buenos Aircs & 56.700 & 44.1 \\
\hline 837 & Green Peas & ," & $"$ & ", & ", & 75.200 & 58.4 \\
\hline 838 & White Peas & ", & , & Mereedes & $"$ & $74 .-$ & 57.5 \\
\hline 839 & Peas & $"$ & ," & Tandil & $"$ & 78.900 & 61.3 \\
\hline 840 & $"$ & José Vienny & & Baradero & $"$ & 78.800 & 61.2 \\
\hline
\end{tabular}




\begin{tabular}{|c|c|c|c|c|c|c|}
\hline \multirow{2}{*}{ No. } & \multirow{2}{*}{ PRODUCT } & \multirow{2}{*}{ EXHIBITOR } & \multicolumn{2}{|c|}{ PLACE OF PRODUCTION } & \multirow{2}{*}{$\begin{array}{c}\text { Kilos } \\
\text { per } \\
\text { Hectolitre }\end{array}$} & \multirow{2}{*}{$\begin{array}{c}\text { Lbs. } \\
\text { per } \\
\text { Bushel } \\
\text { (W'Clls:STrR) }\end{array}$} \\
\hline & & & DISTRICT & PROVINCE & & \\
\hline 841 & Peas & J. Miguens & Cliaseomús & Buenos Aires & 79.500 & 61.7 \\
\hline 842 & White Peas & Sala de Comercio 11 de Sep'bre. & Los Toldos & $"$ & 80.750 & 62.7 \\
\hline 843 & Spanish Peas & G. Lagor & Capital & S. del Estero & 73.800 & 57.3 \\
\hline 844 & Chicharo Pcas & Sala de Comercio 11 de Sep'bre. & Baradero & Buenos Aires & 64.200 & 49.9 \\
\hline 845 & Lentils &, & ," & ," & 76.800 & 59.6 \\
\hline 846 & Lupins & ," & ", &, & 67.200 & 52.2 \\
\hline 847 & Pea-11ut & Juan Müller & Garay & ," & - & - \\
\hline 848 & , & Juan A.de Acevedo Ramos & General Alvarado &, & - & - \\
\hline 849 & Sun Flower & Sala de Comercio 11 de Scp'bre. & ," & ", & 42.100 & 32.7 \\
\hline 850 & ," & Juan $O$. Habegger & Baradero & ," & 36.500 & 28.3 \\
\hline 851 & Castor Seed & Mariano Santillan & Capital & S. del Estero & 55.900 & 43.4 \\
\hline 852 & $"$ & Eseucla de Agricultura & Paraná & Entre Ríos & 54.700 & 4.2 .5 \\
\hline 853 & Yellow Flint Corn & Ricardo Otamendi & Maipú & Buenos dires & 78.400 & 60.9 \\
\hline 854 & , & Alejo Bochatay & Colon & Entre Ríos & 78.400 & 60.9 \\
\hline 855 & ," & Sala de Connercio 11 de Sep'bre. & Soldini & Santa Fé & 78.350 & 60.9 \\
\hline 856 & ," & , & Laboulaye & Córdoba & 78.800 & 61.2 \\
\hline 857 & ," & José Scaldaferri & Capital & Santa Fé & $77 .-$ & 59.8 \\
\hline 858 & ," & Domingo $Z \ddot{i i}$ y Cia, & Baradero & Bucnos Aires & 77.500 & 60.2 \\
\hline 859 & ," & Cárlos Grillini & Del Carril & ", & 80.300 & 62.4 \\
\hline 860 &, & Sala de Comercio 11 de Sep'bre. & Varcla & , & 78.800 & 61.2 \\
\hline 861 &, & , & Casares & , & 79.400 & 61.7 \\
\hline 862 & ," & , & Puan & , & 78.500 & 60.9 \\
\hline 863 & ." & , & Giles & , & $80 .-$ & 62.2 \\
\hline 864 & ," & , & Mareos Paz & ", & 79.200 & 61.5 \\
\hline 865 & ," &, & Liniers &, & 78.300 & 60.9 \\
\hline 866 & ", & ," & General Rodriguez & ,. & 78.600 & 61.- \\
\hline 867 & , & , & Giles & ," & 78.200 & 60.8 \\
\hline 868 & ," & Pedro Fernandez & General Vidal & Buenos Aires & 77.800 & 60.4 \\
\hline 869 & ," & Joaquiu Cursack & Las Colonias & Santa Fé & 74.800 & 58.1 \\
\hline 870 & , & Sala de Comercio 11 de Sep'bre. & General Rodriguez & ", & 77.600 & 60.2 \\
\hline 871 & , & , & Mrcrcedes & ," & 77.200 & $60 .-$ \\
\hline 872 & , & $"$ & Chraeabuco & ", & 79.400 & 61.7 \\
\hline 873 & ," & , & . Pilar & ", & 81.900 & 63.6 \\
\hline 874 & , & , & Mareos Paz & ", & 80.300 & 62.4 \\
\hline 875 & ., & $"$ & Zárate & ", & 78.700 & 61.1 \\
\hline 876 &, & $"$ & Ceneral Rodriguez & " & 79.400 & 61.7 \\
\hline 877 & ," & ", & Lujan & , & 81.700 & 63.5 \\
\hline 878 &, & ," & Záratc & , & 79.200 & 61.5 \\
\hline 879 & $"$ & " & Alberti & " & 78.500 & 60.9 \\
\hline 880 & ", & Luis Perrin & Cor. Vidal & , & 77.800 & 60.4 \\
\hline 881 & $"$ & Olivieri Hnos. & San Pedro & , & 76.700 & 59.5 \\
\hline $88:$ & ," & Jorge Cribbes & Azul & $"$ & 78.200 & 60.8 \\
\hline
\end{tabular}




\begin{tabular}{|c|c|c|c|c|c|c|}
\hline \multirow{2}{*}{ No. } & \multirow{2}{*}{ PRODUCT } & \multirow{2}{*}{$E X H I B I T O R$} & \multicolumn{2}{|c|}{ PLACE OF PRODUCTION } & \multirow{2}{*}{$\begin{array}{c}\text { Kllos } \\
\text { per } \\
\text { Hectolitre }\end{array}$} & \multirow{2}{*}{$\begin{array}{c}\text { Lbs. } \\
\text { per } \\
\text { Bushel } \\
\text { (I'CHESTER) }\end{array}$} \\
\hline & & & DISTRICT & PROVINCE & & \\
\hline 883 & Yellow Flint Corn & Sala de Comcrcio 11 de Sep'bre. & Giles & Buenos Aires & 79.400 & 61.7 \\
\hline 884 & , & $"$ & San Antonio & , & 79.500 & 61.7 \\
\hline 885 & $"$ & $"$ & Las Palmas &, & 78.600 & 61.- \\
\hline 886 & ", & Lorenzo Marcenaro & Rauch &, & 79.200 & 61.5 \\
\hline 887 & ," & Isidro Hnos. & San Pedro &, & $76.800^{\circ}$ & 59.6 \\
\hline 888 & $"$ & Sala de Comercio 11 de Sep'bre. & Casares & ," & 79.300 & 61.6 \\
\hline 889 & ", & $"$ & Chacabuco & ," & 79.300 & 61.6 \\
\hline 890 & $"$ & Jorge Col & Dorrego &, & 78. - & 60.6 \\
\hline 891 & $"$ & Sala de Comcrcio 11 de Sep'bre. & Carlos Kcen & , & 78.800 & 61.2 \\
\hline 892 & , & Juan Basilico & La Capital & Santa Fé & 77.500 & 60.2 \\
\hline 893 & $"$ & Antonio de Llamos & Santa Ana & Misiones & $77 .-$ & 59.8 \\
\hline 894 & ," & Sala de Comercio 11 de Sep'bre. & Lujan & Buenos Aires & 78.300 & 60.9 \\
\hline 895 & $"$ & $"$ & Merlo & $"$ & 77.200 & $60 .-$ \\
\hline 896 &, & $"$ & Irene & ", & $77 .-$ & 59.8 \\
\hline 897 & $"$ & Juan Marcone & San Nicolás &, & 78.100 & 60.7 \\
\hline 898 & ," & Sala de Comercio 11 de Sep'bre. & Necochea & , & 80.100 & 62.2 \\
\hline 899 & , & , & Colonia Apóstoles & Misiones & 79.200 & 61.5 \\
\hline 900 & $"$ & 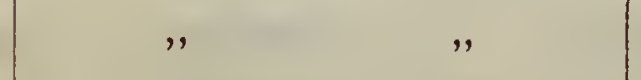 & General Sarmiento & Bucnos Aires & 78.900 & 61.3 \\
\hline 901 & $"$ & $"$ & Pilar & ", & 79.500 & 61.7 \\
\hline 902 & , & , & Casares & , & 78.900 & 61.3 \\
\hline 903 & ," & Jorge Barbich & Baradero & , & 68.400 & 53.1 \\
\hline 904 & ," & Pastor Senillosa & Ayacucho & $"$ & 79.900 & 62.1 \\
\hline 905 & , & Porta Hnos. & San Pedro & , & 76.500 & 59.5 \\
\hline 906 & White Flint Corn & Pastor Senillosa & Ayacucho & ," & 77. - & 59.8 \\
\hline 907 & , & Sala de Comercio 11 de Sep'bre. & - & Córdoba & $81 .-$ & 63.- \\
\hline 908 & , & $"$, & Carlos Kecn & Buenos Aires & 82.100 & 63.7 \\
\hline 909 &, & B. Sampurein & Las Flores &, & 79.900 & 62.1 \\
\hline 910 & , & F. Maneto & Chacabuco & , & $79 .-$ & 61.4 \\
\hline 911 & , & Mateo Dorado & Capital & S. del Estero & 79. -1 & 61.4 \\
\hline 912 & , & Jullo A. Barceló & Rauch & Buenos Aires & 77.4 .00 & 60.2 \\
\hline 913 & , & Sala de Comercio 11 de Sep'bre. & Lujan & , & 82. - & 63.6 \\
\hline 914 & $"$ & , , , & Carlos Kecn & , & 81. - & 63.- \\
\hline 915 & , & $"$ & T. Suarez & , & 80.500 & 62.5 \\
\hline 916 & , &, & Carlos Keen & , & 80.700 & 62.7 \\
\hline 917 & , & F. Castex Madarriaga & Tandil & ,. & 79.100 & 61.5 \\
\hline 918 & , & Miguel Hidoz & Rauch & , & 79.400 & 61.7 \\
\hline 919 & , & Tomas Martearena & Castelli & , & 79.500 & 61.7 \\
\hline 920 & ", & Patricio B. Dillon & S. Andres de Giles & , & 80. - & 62.2 \\
\hline 921 & $"$ & Felix Moreno & Ojo de Agua & S. del Estero & 79.800 & 62.- \\
\hline 922 & $"$ & A. Alsina & Baradero & Buenos Aires & 79.600 & 61.8 \\
\hline 923 & White Dent Corn &, & , & $"$ & 76.200 & 59.2 \\
\hline 924 & " & Luis Icuzzi & Reconquista & Santa Fé & 72.500 & 51.3 \\
\hline
\end{tabular}




\begin{tabular}{|c|c|c|c|c|c|c|}
\hline \multirow{2}{*}{ No. } & \multirow{2}{*}{ PRODUCT } & \multirow{2}{*}{$E X H I B I T O R$} & \multicolumn{2}{|c|}{ PLACE OF PRODUCTION } & \multirow{2}{*}{$\begin{array}{c}\text { Kilos } \\
\text { per } \\
\text { Hectolitre }\end{array}$} & \multirow{2}{*}{$\begin{array}{c}\text { Lbs. } \\
\text { per } \\
\text { Bushel } \\
\text { (W'CHESTER) }\end{array}$} \\
\hline & & & DISTRICT & PROVINCE & & \\
\hline 925 & Chiriguano Corn & P. Chavarria & Candelaria & Salta & 63.700 & 49.5 \\
\hline 926 & Cateto Corn & Sala de Comercio 11 de Sep'bre. & Colonia Apóstoles & Misiones & 67.600 & 52.5 \\
\hline $92 \pi$ & Pop Corn & Cruz Lucero & Río $4^{\circ}$ & Córdoba & 79.900 & 62.1 \\
\hline 928 & , & Andres Martinelli & Colon & ", & 78.800 & 61.2 \\
\hline 929 & ," & Sala de Comercio 11 de Sep'bre & - & " & 80.300 & 62.4 \\
\hline 930 & Sweet Corn &,$\quad$, & - & Buenos Aires & 63.250 & 49.2 \\
\hline 931 & $"$ & Lorenzo Marcenaro & Rauch & , & $64 .-$ & 49. 7 \\
\hline 932 & Yellow Flint Corn & Sala de Comercio 11 de Sep'bre. & Alvear & Santa Fé & 77.300 & 60.1 \\
\hline 933 & $"$ & , , , & C. Keen & Buenos Aires & 80.100 & 62.2 \\
\hline 934 & $"$ & " $\quad, \quad \quad "$ & Alvarez & Santa Fé & 78.4 .00 & 60.9 \\
\hline 935 & Flour 00 & Antonio Battilana & City of & Buenos Aires & - & - \\
\hline 936 & , & R. Oneto y Cia. & ", & , & - & - \\
\hline 937 & " & Enrique y Eduardo Battilana & ", & ," & - & - \\
\hline 938 & , & Oneto y Gallino & $"$ & $"$ & - & - \\
\hline 939 & , & Ceriale, Viggiolo y Cia. & , & $"$ & - & - \\
\hline 940 & , & Juan C. Bancalari y Cia. & $"$ & " & - & - \\
\hline 941 & ", & Del Piano y Lucas & " & ", & - & - \\
\hline 942 & , & Castagnino y Fulchi & $"$ & ," & - & - \\
\hline 943 &, & Miguel Oneto & $"$ & , & - & - \\
\hline 944 & Flour 0 & R. Oneto y Cia. & " & , & - & - \\
\hline 945 & , & E. y E. Battilana & $"$ & $"$ & - & - \\
\hline 946 &, & Oneto y Gallino & $"$ & , & - & - \\
\hline 947 & , & Ceriale, Viggiolo y Cia. & $"$ & $"$ & - & - \\
\hline 948 & , & Juan C. Bancalari y Cia. & $"$ & $"$ & - & - \\
\hline 949 & , & Del Piano y Lucas & , & $"$ & - & - \\
\hline 950 &, & Castagnino y Fulchi & , & $"$ & - & - \\
\hline 951 & , & Miguel Oneto & , & , & - & - \\
\hline 952 & Flour 00 & Usanna y Badnio & $"$ & ", & - & - \\
\hline 953 & , & Molino Argentino & $"$ & $"$ & - & - \\
\hline 954 & Flour 0 & Usanna y Badino & $"$ & ", & - & - \\
\hline 955 & Flour $1^{\text {st }}$ & Antonio Battilana & ", & ", & - & - \\
\hline 956 & $"$ & R. Oneto y Cia. & ", & ", & - & - \\
\hline 957 & , & E. y E. Battilana & ", & " & - & - \\
\hline 958 & ", & Oneto y Gallino &, & " & - & - \\
\hline 959 &, & Ceriale, Viggiolo y Cia. & ", & $"$ & - & - \\
\hline 960 &, & Juan C. Bancalari y Cia. & $"$ & ", & - & - \\
\hline 961 & ,. & Del Piano y Lucas & $"$ & " & - & - \\
\hline 962 &, & Castagnino y Fulchi & $"$ & $"$ & - & - \\
\hline 963 & , & Miguel Onetto & , & $"$ & - & - \\
\hline 964 & $"$ & Usanna y Badino & $"$ & ", & - & - \\
\hline 965 & Flour $2^{\text {nd }}$ & R. Oneto y Cia. & $"$ & $"$ & - & - \\
\hline 966 & $"$ & E. y E. Battilana & $"$ & , & - & - \\
\hline
\end{tabular}




\begin{tabular}{|c|c|c|c|c|c|c|}
\hline \multirow{2}{*}{ No. } & \multirow{2}{*}{ PRODUCT } & \multirow{2}{*}{$E X H I B \mid T O R$} & \multicolumn{2}{|c|}{ PLACE OF PRODUCTION } & \multirow{2}{*}{$\begin{array}{c}\text { Kilos } \\
\text { per } \\
\text { Hectolitre }\end{array}$} & \multirow{2}{*}{$\begin{array}{l}\text { Lbs. } \\
\text { per } \\
\text { Bushel } \\
\text { (W'CHESTRR) }\end{array}$} \\
\hline & & & DISTRICT & PROVINCE & & \\
\hline 967 & Flour $2^{\text {mil }}$ & Oneto y Gallino & City of & Buenos Aires & - & - \\
\hline 968 & , $1^{\text {st }}$ & Ccriale, Viggiolo y Cía. & , & , & - & - \\
\hline 969 & ." & Juan C. Bancalari y Cía. & ", &, & - & - \\
\hline 970 &, & Del Piano y Lucas. & , & ," & - & - \\
\hline 971 &, & Molino Oneto & , & ," & - & - \\
\hline 972 & $"$ & Migucl Oneto & , & ," & - & - \\
\hline 973 &, & Usanna y Badino & $"$ & ", & - & - \\
\hline 974 & Pollard & Antonio Batilana & $"$ & ", & - & - \\
\hline 975 &, & R. Oneto y Cía. & , & " & - & - \\
\hline 976 &, & E. y E. Batilana & , & ," & - & - \\
\hline 977 & ," & Oneto y Gallino & , & " & - & - \\
\hline 978 & , & Cerialc, Viggiolo y Cía. & ." & , & - & - \\
\hline 979 & , & Juan C. Bancalari y Cía. & $"$ & ", & - & - \\
\hline 980 & , & Del Piano y Lucas & $"$ & " & - & - \\
\hline 981 &, & Castagnino y Fulchi & " & " & - & - \\
\hline 982 & ," & Miguel Oneto & " & ," & - & - \\
\hline 983 & , & Usanna y Badino & $"$ & ,. & - & - \\
\hline $9 \$ 4$ & Semola & Miguel Oneto & , & ", & - & - \\
\hline 985 & Sharps & Antonio Batilana & , & , & - & - \\
\hline 986 & , & E. y E. Batilana & , & , & - & - \\
\hline 987 & , & Oncto y Gallino & " & , & - & - \\
\hline 988 & $"$ & Cerialc, Viggiolo y Cía. &, & ", & - & - \\
\hline 989 & $"$ & Juan C. Bancalari y Cía. & , & " & - & - \\
\hline 990 & , & Del Piano y Lucas &, & , & - & - \\
\hline 991 & ", & Castagnino y Fulchi & , & $"$ & - & - \\
\hline 992 & , & Miguel Oneto & , & ," & - & - \\
\hline 993 & $"$ & Usanna y Badino & , & , & - & - \\
\hline 994 & Bran & Antonio Battilana & " & , & - & - \\
\hline 995 &, & R. Oneto y Cía. & $"$ &, & - & - \\
\hline 996 & , & E. $y$ E. Batilana &, & $"$ & - & - \\
\hline $99 \pi$ & $"$ & Oneto y Gallino & " & " & - & - \\
\hline 998 & , & Ceriale Viggiolo y Cía. & , & $"$ & - & - \\
\hline 999 & $"$ & Juan C. Bancalari y Cía. &, & ," & - & - \\
\hline 1000 & $"$ & Del Pino y Lucas & ,, & ," & - & - \\
\hline 1001 & ," & Castagnino y Fulchi & , & , & - & - \\
\hline 1002 &, & Miguel Oncto & , & , & - & $一$ \\
\hline 1003 & , & Usanna $y$ Badino & ," & ", & - & - \\
\hline 1004 & Barletta Wheat & Felix Raimundi & San Gcrónimo & Santa Fé & 84. - & 65.3 \\
\hline 1005 & $"$ & Felix Peralta & , & , & 83.450 & 64.8 \\
\hline 1006 & ", & Zaverza y Duplat & Saavedra & Bucnos Aires & 84.150 & 65.4 \\
\hline 1007 & $"$ & José Broggi & Pehuajó & $"$ & 84.500 & 65.6 \\
\hline 1008 & ," & Carlos Senino & Sampacho & Córdoba & 85.150 & 66.2 \\
\hline
\end{tabular}




\begin{tabular}{|c|c|c|c|c|c|c|}
\hline \multirow{2}{*}{ No. } & \multirow{2}{*}{ PRODUCT } & \multirow{2}{*}{$E X H|B| T O R$} & \multicolumn{2}{|c|}{ PLACE OF PRODUCTION } & \multirow{2}{*}{$\begin{array}{c}\text { Kilos } \\
\text { per } \\
\text { Hectolitre }\end{array}$} & \multirow{2}{*}{$\begin{array}{c}\text { Lbs. } \\
\text { per } \\
\text { Bushel } \\
\text { (W'CnesTER) }\end{array}$} \\
\hline & & & DISTRICT & PROVINCE & & \\
\hline 1009 & Barletta Wheat & Angel Medici & Iriondo & Santa Fé & 82.200 & 63.8 \\
\hline 1010 & , & Gianello y Bettarel & Gualeguaychú & Entre Ríos & 82.550 & 64.1 \\
\hline 1011 & ," & Sauzzone Hnos. & Rojas & Buenos Aires & 81.150 & 63.- \\
\hline 1012 &, & Ledesma Hnos. & San Gerónimo & Santa Fé & 81.400 & 63.2 \\
\hline 1013 & " & Ramón Zafille & Unión & Córdoba & 83.100 & $6+.6$ \\
\hline 1014 & , & Bartolo Zabena & San Martin & Santa Fé & 82.550 & 64.1 \\
\hline 1015 & ", & Luis Pasquetti & San Lorenzo & , & 81.400 & 63.2 \\
\hline 1016 & , & Cayetano Negri & Nogoyá & Entre Ríos & 83.700 & $65 .-$ \\
\hline 1017 & " & Viuda de Soldaracen é hijos & Gualeguaychú & $"$ & 84. - & 65.3 \\
\hline 1018 & ", & Juan T. Laurcuce & Villarino & Butenos Aires & 83.900 & 65.2 \\
\hline 1019 & ," & Francisco Arece & Adolfo Alsina & ", & 83.100 & 64.6 \\
\hline 1020 &, & F.J.Jeonney & ", &, & 83.550 & 64.9 \\
\hline 1021 & $"$ & Sucesión Diego Merlo & Trenque Lauquen & , & 82.050 & 63.7 \\
\hline 1022 & ," & Capoduro Hnos. & Belgrano & Santa Fé & 83.350 & 64.7 \\
\hline 1023 & ", & Juan Bock & La Capital &, & 81.050 & 63.- \\
\hline 1024 & , & Augusto Bohl & Unión & Córdoba & 82.750 & 64.3 \\
\hline 1025 & , & G. Giraud & Colonia Piaggio & Santa Fé & 83.350 & 64.7 \\
\hline 1026 & , & Luis Nozeda & La Capital & $"$ & $\$ 3.550$ & 64.9 \\
\hline 1027 & $"$ & Pedro Luro é hijos & Villarino & Buengs Aires & 81.400 & 63.2 \\
\hline 1028 & $"$ & Gedds Hnos. & Coronel Pringles & , & 83.450 & $6+.8$ \\
\hline 1029 & $"$ & Pedro Buron & , &, & 83.800 & 65.1 \\
\hline 1030 & $"$ & Ignacio Aspinoz & , & , & 83.450 & 64.8 \\
\hline 1031 & " & Maria Daniele de Rosmo & San Gerónimo & Santa Fé & 83.900 & 65.2 \\
\hline 1032 & , & Cárlos Semino & Coroncl Bustos & Córdoba & 84.600 & 65.7 \\
\hline 1033 & ," & Vicente Pericas & General Lopez & Santa Fé & 83.100 & 64.6 \\
\hline 1034 & " & Mariano Lopez & Bragado & Buenos Aires & 83.200 & 64.6 \\
\hline 1035 & , & José Soldati & General Pinto & , & 84.750 & 65.4 \\
\hline 1036 & , & Nacinveni Hnos. & S. Andres de Giles &, & 82.200 & 63.8 \\
\hline 1037 & $"$ & Colastiné de Ledesma & San Gerónimo & , & $83 .-1$ & 64.5 \\
\hline 1038 & ", & Perna y Romagnoli & General Villegas & ," & 83.4 .50 & 64.8 \\
\hline 1039 & , & Stuckey y Gerber & Río $3^{\circ}$ Arriba & Córcloba & 83.350 & 64.7 \\
\hline 1040 & $"$ & Pedro Bazzanelle & Bahia Blanca & Buenos Aires & 83.550 & 64.9 \\
\hline 1041 & , & José Pierrot & Iriondo & Santa Fé & $\$ 2.200$ & 63.8 \\
\hline 1042 & Arrieta Wheat & Pablo Massoni & Lujan & Buenos Aires & 81.850 & 63.6 \\
\hline 1043 & $"$ & Carlos Barbcro & Matanza & , & 82.050 & 63.7 \\
\hline 1044 & $"$ & B. P. Balvidanes & Trenque Lauquen & ", & 80.550 & 62.6 \\
\hline 1045 & , & Felipe S. Weiner & Las Colonias & Santa Fé & 81.950 & 63.6 \\
\hline 1046 & $"$ & Mina Hnos. & Castellanos & , & 81.150 & 63.- \\
\hline 1047 &, & Bartolino Hnos. & San Martin & $"$ & 84.250 & 65.5 \\
\hline 1048 & $"$ & Humberto Elliff & Chacabuco & Buenos Aires & 80.700 & 62.7 \\
\hline 1049 & , & Alberto Ortiz & Chivilcoy & , & 82.650 & 64.2 \\
\hline 1050 & Russian Wheat & Perez Hnos. & Coronel Dorrego & ," & 81.500 & 63.3 \\
\hline
\end{tabular}




\begin{tabular}{|c|c|c|c|c|c|c|}
\hline \multirow{2}{*}{ No. } & \multirow{2}{*}{ PRODUCT } & \multirow{2}{*}{$E X H|B| T O R$} & \multicolumn{2}{|c|}{ PLACE OF PRODUCTION } & \multirow{2}{*}{$\begin{array}{c}\text { Kilos } \\
\text { per } \\
\text { Hectolitre }\end{array}$} & \multirow{2}{*}{$\begin{array}{c}\text { Lbs. } \\
\text { per. } \\
\text { Bushel } \\
\text { (h'chester) }\end{array}$} \\
\hline & & & DISTRICT & PROVINCE & & \\
\hline 1051 & Russian Wheat & Humberto Elliff & Chivilcoy & Buenos Aires & 83. - & 64.5 \\
\hline 1052 & $"$ & Miguel Aberasturi & Coronel Dorrego & , & 80.100 & 62.2 \\
\hline 1053 & , & José Broggi & Pehuajó & , & 83.900 & 65.2 \\
\hline $10 \overline{5}$ & $"$ & José Daga & Caseros & Santa Fé & 82.200 & 63.8 \\
\hline 1055 & , & Humberto Elliff & Chacabuco & Buenos Aires & 82.650 & 64.2 \\
\hline 1056 & , & Guillermo Lett & A. Alsina & $"$ & 79.200 & 61.5 \\
\hline 1057 & ", & Felipe Crosetti & Belgrano & Santa Fé & 84.500 & 65.6 \\
\hline 1058 & ," & Celedonio Pereda & Trenque Lauquen & Buenos Aires & 80.900 & 62.9 \\
\hline 1059 & Italian Wheat & Cayetano Negri & Nogoyá & Entre Ríos & 82.650 & 64.2 \\
\hline 1060 & , & Bautista Formento & Paraná & , & $\$ 1.150$ & 63.- \\
\hline 1061 & $"$, & Pedro Bartolo & Río 3.० Arriba & Córdoba & 83.450 & 64.8 \\
\hline 1062 & Lombardy Wheat & Alejo Bochatoz & Colon & Entre Ríos & 79.450 & 61.7 \\
\hline 1063 & French Wheat & Isidro E. Loustouret & Saladillo & Buenos Aires & 81.250 & 63.1 \\
\hline 1064 & " & F. Budin & Unión & Córdoba & 82.850 & 64.4 \\
\hline 1065 & $"$ & Mariano López & Braga & Buenos Aires & 82.200 & 63.8 \\
\hline 1066 & ," & Antonio Soler & Lamadrid & " & 78.350 & 60.9 \\
\hline 1067 & ", & Alberto Ortiz & Chivilcoy & ," & 78.750 & 61.2 \\
\hline 1068 & , & José Abadié & Coronel Pringles & ", & 80.900 & 62.9 \\
\hline 1069 & $"$ & Bartolino Hnos. & San Martin & Santa Fé & 83.450 & 64.8 \\
\hline 1070 & Hungarian Wheat & Rómulo Franco & Trenque Lauquen & Buenos Aires & 81.700 & 63.5 \\
\hline 1071 & Candeal Wheat & Félix Bartomer & 25 dc Мayo & , & 82.050 & 63.7 \\
\hline 1072 & , & Julio Bouchard & Gualeguaychú & Entre Ríos & 82.850 & 64.4 \\
\hline 1073 & ", & Bolsa dc Cereales & Rosario & Santa Fé & 83.350 & 64.7 \\
\hline 1074 & " & Esteban Garré & Trenque Lauquen & Buenos Aires & 82.650 & 64.2 \\
\hline 1075 & ," & D. Luna & Loreto & S. del Estero & 80.350 & 62.4 \\
\hline 1076 & Anchuelo Wheat & Cayetano Negri & Nogoya & Entre Ríos & 83.450 & 64.8 \\
\hline 1077 & ., & Gianello y Betarel & Gualeguay & , & 80. - & 62.2 \\
\hline 1078 & ." & W. Goldaracena & Gualeguaychú & , & 84.350 & 65.5 \\
\hline 1079 &, & Agustín Barbati & San Cristóbal & Santa Fé & 82.650 & 64.2 \\
\hline 1080 & Chubut Wheat & Rascaugnet Hnos. & Tandil & Buenos Aires & 80.350 & 62.4 \\
\hline 1081 & Russian Wheat & Bolsa de Cereales & Rosario & Santa Fé & 82.400 & $64 .-$ \\
\hline 1082 & White Wheat & A. González Moyano & Copo $1 .^{\circ}$ & S. dej Estero & 80.550 & 62.6 \\
\hline 1083 & Linseed & Celeste Romagnoli & General Villegas & Buenos Aires & 66.500 & 51.7 \\
\hline 1084 & , & Bautista Giudia & Iriondo & Santa Fé & 67.800 & 52.7 \\
\hline 1085 & ", & Angel Midia & $"$ & $"$ & 69.850 & 54.3 \\
\hline 1086 & , & Federico Lurig & La Capital & $"$ & 67.900 & 52.8 \\
\hline 1087 & ," & Luis Cedermay & Paraná & Entre Ríos & 69.100 & 53.7 \\
\hline 1088 &. & Viuda de Soldaracena é hijos & Gualeguaychú & ", & 71.600 & 55.6 \\
\hline 1089 & , & José Carrera & Chivilcoy & Buenos Aires & $67 .-$ & 52.1 \\
\hline 1090 & " " & Anacleto Borattini & Rojas & , & 67.500 & 52.5 \\
\hline 1091 & , & Manuel Martínez & Marcos Paz & , & 68.400 & 53.1 \\
\hline 1092 & , & Estancia Company & San Martin & Santa Fé & 67.500 & 52.5 \\
\hline
\end{tabular}




\begin{tabular}{|c|c|c|c|c|c|c|}
\hline \multirow{2}{*}{ No. } & \multirow{2}{*}{ PRODUCT } & \multirow{2}{*}{ EXHIBITOR } & \multicolumn{2}{|c|}{ PLACE OF PRODUCTION } & \multirow{2}{*}{$\begin{array}{c}\text { Kilos } \\
\text { per } \\
\text { Hectolitre }\end{array}$} & \multirow{2}{*}{$\begin{array}{c}\text { Lbs. } \\
\text { per } \\
\text { Bushel } \\
\text { (w'rnisster) }\end{array}$} \\
\hline & & & DISTRICT & PROVINCE & & \\
\hline 1093 & Linseed & Francisco Francioni & San Martin & Santa Fé & 68.700 & 53.4 \\
\hline 1094 & , & Nacinoveni Hnos. & San Andrés de Giles & Buenos Aires & 70.400 & 54.7 \\
\hline 1095 & , & Marcone Hnos. & San Nicolás & , & 71.900 & 55.8 \\
\hline 1096 & Neapolitan Linseed &. & , & ", & 68.500 & 53.2 \\
\hline 1097 & Linseed & Bautista Giuliano & General Lopez & Santa Fć & 69.800 & 54.3 \\
\hline 1098 & , & Bartolino Hnos. & San Martin &, & 68.500 & 53.7 \\
\hline 1099 & Barley & Julio A. Barceló & Rauch & Buenos Aires & 60.050 & 46.7 \\
\hline 1100 & , & Adolfo Quintana & Javi & Jujuí & 63.800 & 49.6 \\
\hline 1101 & , & Emilio Caurrieres. & - & Catamarca & 63. - & 48.9 \\
\hline 1102 & , & Semino Hnos. & - & Santa Fé & 63.500 & 49.3 \\
\hline 1103 & Malting Barley & Sala de Comercio 11 de Sep'bre. & Puan & Buenos Aires & 64.600 & 50.2 \\
\hline 1104 & , $\quad$, & ", $\quad$, & Berutti & , & 68.900 & 53.5 \\
\hline 1105 & Oats & Juan Bursa & Guaminí & , & 49.850 & 38.7 \\
\hline 1106 &, & N. G. Noboa & Saladillo & , & 4.1 .300 & 32.1 \\
\hline 1107 & $"$ & Casiano Vieytes & Coronel Pringles & ," & 53.200 & 41.3 \\
\hline 1108 & , & Sala de Comercio 11 de Sep'bre. & G. Chaves & , & 52.4 .00 & 41.5 \\
\hline 1109 & ", & Juan . . Alberdi & Coronel Suarez & , & 47.300 & 36.7 \\
\hline 1110 & , & Juan N. Barreneche & Gencral Lamadrid & " & 50.150 & 39.- \\
\hline 1111 & $"$ & Enilio Conmeres & Pilar & , & 44.200 & $3+.3$ \\
\hline 1112 & , & Nogueras y Avalos & F. Madero & , & 48.250 & 37.5 \\
\hline 1113 & $"$ & " " " & ", & $"$ & 52.350 & 40.7 \\
\hline 1114 & ," & P. V. Jauregui & Bayauca &, & 46.600 & 36.2 \\
\hline 1115 & " & Nogueras y Avalos & F. Madero &, & 54.150 & 42.1 \\
\hline 1116 & , & Devoto, Balbiani y Cia. & Olascoaga & ," & 45.450 & 35.3 \\
\hline 1117 & ," & Ojea y García & San Antonio &, & 41.800 & 32.5 \\
\hline 1118 & ,. & Marcone Hnos. & San Nicolás &, & 43.900 & 34.1 \\
\hline 1119 & , & G. I'értora & Kanki & Pampa Centr. & 45.450 & 35.3 \\
\hline 1120 & , & Pedro Dasoli & Giles & Buenos dires & 49.650 & 38.6 \\
\hline 1121 & , & Bolas de Cereales & Rosario & Santa Fé & 52.500 & 40.8 \\
\hline 1122 & , & G. Lértora & Kanki & Pampa Centr. & 41.300 & 32.1 \\
\hline 1123 & , & Ojea y Garcia & San Antonio & Buenos Aires & 46.500 & 36.1 \\
\hline 1124 & , & Juan Rebalfo & Bragado & , & 43.900 & 34.1 \\
\hline 1125 & , & E. Greslebin & $25 \cdot$ de Mayo & ," & $47-$ & 36.5 \\
\hline 1126 & ," & S. Unzué é hijos & San Emilio & , & 46.300 & $36 .-$ \\
\hline 1127 & Rye & S. Batestrasse & Junin & " & 69.300 & 53.8 \\
\hline 1128 & , & Emilio D. Ortiz & San Martin & , & 75.550 & 58.7 \\
\hline 1129 & ", & W. Buzzoni & Belgrano & , & 73.850 & 57.4 \\
\hline 1130 & Canary seed & Julio Maxit & Colon & Entre Ríos & 75.400 & 58.6 \\
\hline 1131 & , & Marcone Hnos. & San Nicolás & Buenos Aires & 73.800 & 57.3 \\
\hline 1132 & , & Orcoyen Castañas y Co. & Rojas & , & 73.900 & 57.4 \\
\hline 1133 & Sugar-Cane & Esscuela de Agricultura & Paraná & Entre Ríos & 73.600 & 57.1 \\
\hline 1134 & Linseed & Juaı Albizú & Laprida & Buenos Aires & 68.700 & 53.4 \\
\hline
\end{tabular}




\begin{tabular}{|c|c|c|c|c|c|c|}
\hline \multirow{2}{*}{ No. } & \multirow{2}{*}{ PRODUCT } & \multirow{2}{*}{$E X H|B| T O R$} & \multicolumn{2}{|c|}{ PLACE OF PRODUCTION } & \multirow{2}{*}{$\begin{array}{c}\text { Kilos } \\
\text { per } \\
\text { Hectolitre }\end{array}$} & \multirow{2}{*}{$\begin{array}{c}\text { Lbs. } \\
\text { per } \\
\text { Bushel } \\
\text { (W'CHESTER) }\end{array}$} \\
\hline & & & DISTRICT & PROVINCE & & \\
\hline 1135 & Linseed & Juan Verna & General Villegas & Buenos Aires & 66.200 & 51.5 \\
\hline 1136 & " & Fabiano Chieroni & La Capital & Santa Fé & 68.800 & 53.5 \\
\hline 1137 & ", & F. Riveras & Záratc & Buenos Aires & 69.800 & 54.2 \\
\hline 1138 & " & Colonia Piaggio & San Gerónimo & Santa Fé & 67.400 & 52.3 \\
\hline 1139 & $"$ & I tuis Gnanzirotti & Guamini & Buenos Aires & 64.800 & 50.3 \\
\hline 1140 & , & R. S. Coelho & Constitución & Santa Fé & 69.200 & 53.7 \\
\hline 114.1 &, & Augusto Bohl & Unión & Córdoba & 66. - & 51.3 \\
\hline 1142 & , & Mina Hnos. & Castellanos & Santa Fé & 68. - & 52.9 \\
\hline 1143 & $"$ & Roberto Hope & San Lorenzo & " & 67.300 & 52.2 \\
\hline 1444 & ," & Francisco Ridiero & Caseros & $"$ & 70.600 & 54.8 \\
\hline $114 \overline{5}$ & $"$ & Gaitan Vidal & Zárate & Buenos Aires & 69.700 & 54.1 \\
\hline 1146 & ", & Estancia "Los Corrales" & Las Colonias & Santa Fé & 67.700 & 52.6 \\
\hline 1147 & ," & José Fondevila & General Belgrano & Buenos Aires & 66.200 & 51.4 \\
\hline 1148 & Turnip-seed & Bolsa de Cereales & Rosario & Santa Fé & 68.500 & 53.2 \\
\hline 1149 & $"$ & Velland $y$ Rodriguez & Colón & Entre Ríos & 66.300 & 51.5 \\
\hline $11 \tilde{5} 0$ & ", & Miguel Angel Dimario & Balcarce & Buenos Aires & 62.500 & 48.6 \\
\hline 1151 & ", & Alfrecto Rousseau & Iriondo & Santa Fé & 64. - & 49.7 \\
\hline 1152 & ,. & Orcoyen, Castaños y Co. & Acevedo & Buenos Aires & 66.600 & 51.7 \\
\hline 1153 & ," & F. Saforcada & Junin & , & 66.900 & $52 .-$ \\
\hline 1154 & , & A. Velaz y Co. & Mazanares & , & 65.600 & $51 .-$ \\
\hline $115 \overline{5}$ & , & Carlos Penatti & Nogoyá & Entre Ríos & 67.100 & 52.2 \\
\hline 1156 & ", & J. R. Ramsay & Saforcada & Buenos Aires & 66.100 & $51 . t$ \\
\hline $115 \tau$ & Mustard-seed & Bolsa de Cereales & Rosario & Santa Fé & 66.700 & 51.8 \\
\hline 1158 & , & E. Purcinelli & , & , & 68.200 & $53 .-$ \\
\hline 1159 & Pea - Nut & Vicente Alonso & Las Garzas & ", & - & - \\
\hline 1160 & $", \quad$, & Agustin Malleres & Concordia & Entre Ríos & - & - \\
\hline 1161 & Kidney Beans & Adolfo Quintana & Javí & Jujuy & 62.300 & 48.4 \\
\hline 1162 & Beans & Juan José Castellanos & Candelaria & Salta & 76.500 & 59.5 \\
\hline 1163 & " & Benito Trefo & Loreto & S. del Estero & 78.500 & $61 .-$ \\
\hline $116+$ & Lentils & Bolsa de Cereales & Rosario & Santa Fé & 76.100 & 59.1 \\
\hline $116 \overline{0}$ & Lucern & Julio Maxit & Colón & Entre Ríos & (1) & - \\
\hline 1166 & ", & Federico W'ulff & Azul & Buenos Aires & (2) & - \\
\hline 1167 & , & Pio Ferrarese & J. Celman & Córdoba & (3) & $\dot{-}$ \\
\hline 1168 & " & Juan Ambroggio & M. Juarez & $"$ & (4) & - \\
\hline 1169 & Cotton & Marcos Briolini & Colonia Benitez & Chaco & - & - \\
\hline 1170 & , & $" \quad$, & ", &. & - & - \\
\hline 1171 & ," & Emilio Molas & Piedra Blanca & Catamarca & - & - \\
\hline 1172 & Cotton seed & Marcos Briolini & Colonia Benitez & Chaco & - & - \\
\hline 1173 & Cotton in pod & Claudio Zian & Reconquista & Santa Fé & - & - \\
\hline 1174 & White Wheat & Sala de Comercio 11 de Sep'bre. & - & Buenos Aires & 80.350 & 62.4 \\
\hline 1175 & Barletta & Perez Hnos. & Gencral Dorrego & " & 78.550 & $61 .-$ \\
\hline 1176 & " & Marcone Hnos. & San Nicolás & $"$ & 81.150 & 63.- \\
\hline
\end{tabular}

(1) Weight absolute $2200 \mathrm{mg}$ - - (2) Weight absolnte $2120 \mathrm{mg}$. - (3) Weight absolute $2000 \mathrm{mg}$. - (4) Weight absolute $1985 \mathrm{mg}$. 


\begin{tabular}{|c|c|c|c|c|c|c|c|}
\hline \multirow{2}{*}{ No. } & \multirow{2}{*}{ PRODUCT } & \multirow{2}{*}{\multicolumn{2}{|c|}{ EXHIBITOR }} & \multicolumn{2}{|c|}{ PLACE OF PRODUCTION } & \multirow{2}{*}{$\begin{array}{c}\text { Kilos } \\
\text { per } \\
\text { Hectolitre }\end{array}$} & \multirow{2}{*}{$\begin{array}{c}\text { Lbs. } \\
\text { per } \\
\text { Bushel } \\
\text { (II'CuESTER) }\end{array}$} \\
\hline & & & & DISTRICT & PROVINCE & & \\
\hline 1177 & Barletta Wheat & Cesa & re Gieco & Diamante & Entre Rios & 80.800 & 62.8 \\
\hline 1178 & ", & Jorge $\mathrm{C}$ & Gschwind & San Martin & Santa Fé & 84. - & 6.5 .3 \\
\hline 1179 & $"$ & Sant & a Anita & ", & ", & 83.550 & 64.9 \\
\hline 1180 & $"$ & Francis & co Ridiero & Caseros & ", & 84.150 & 65.4 \\
\hline 1181 & Pea-Nut & Juan & Marega & Reconquista & ,. & - & - \\
\hline 1182 & Medeah Wheat & Escuela de & Agricultura & Villa Constitución & $"$ & - & - \\
\hline 1183 & Barletta Wheat & . & $"$ & , & $"$ & - & - \\
\hline 1184 & $" \quad "$ & , , & $"$ & $"$ & $"$ & - & - \\
\hline 1185 & " " " & ", , & , & , & $"$ & - & - \\
\hline 1186 & Fucense Wheat & $", \quad$, & ,. & ", &, & - & - \\
\hline 1187 & Rieti Wheat & , , , & $"$ &, & , & - & - \\
\hline 1188 & American Wheat &.,$\quad$, & $"$ & ", & " & - & - \\
\hline 1189 & Rye & $" \quad "$ & $"$ & ,. & ," & - & - \\
\hline 1190 & Chevalier Wheat & " & $"$ & $"$ & $"$ & - & - \\
\hline 1191 & Oats & $" \quad "$ & $"$ & $"$ & ," & - & - \\
\hline 1192 & Linseed & $"$, & , & , & , & - & - \\
\hline 1193 & $"$ & $"$, & , & ", & $"$ & - & - \\
\hline 1194 & Lucern & Celestino & Fernandez & Gral. Villegas & Buenos Aires & - & - \\
\hline 1195 & White Maize & Horacio & P. Carreras & Various & ," & (1) & - \\
\hline 1196 & " " & , & $"$ & $"$ & ", & $(2)$ & - \\
\hline 1197 & Yellow Maize & , & , & $"$ & ." & (3) & - \\
\hline 1198 & French Wheat & , & ", & $"$ & ". & (4) & - \\
\hline 1199 & Barletta Wheat & , & , & Bolivar & ", & (5) & - \\
\hline 1200 & Flour & $"$ & , & Pancalari & , & (6) & - \\
\hline 1201 & $"$ & " & $"$ & $"$ & , & (T) & - \\
\hline 1102 & Fine corn meal & $"$, & $"$ & - & , & $(s)$ & - \\
\hline
\end{tabular}

11 -. Preserved by the "Carreras" process. 
